\title{
Polyrotaxane and Polyrotaxane Network: Supramolecular Architectures Based on the Concept of Dynamic Covalent Bond Chemistry
}

\author{
Toshikazu TAKATA $^{\dagger}$ \\ Department of Organic and Polymeric Materials, Tokyo Institute of Technology, \\ 2-12-1 Ookayama, Meguro-ku, Tokyo 152-8552, Japan
}

(Received November 2, 2005; Accepted November 7, 2005; Published January 15, 2006)

\begin{abstract}
This review article concerns with the syntheses of polyrotaxanes and polyrotaxane networks that can be constructed mainly on the basis of the concept of dynamic covalent bond chemistry. At the beginning of the review, synthetic methods of rotaxanes are briefly summarized along with several high yielding preparations. Synthesis of poly[3] rotaxane by the reaction of homoditopic monomers utilizing the thiol-disulfide interchange reaction was discussed in detail, among polyrotaxanes possessing topological bonds for the monomer linking in the main chain. Polyrotaxane network was prepared by a similar protocol from a polyfunctional crown ether and a dumbbell-shaped homoditopic axle containing two sec-ammonium salt moieties and centrally located disulfide bond. Some characteristics of the polyrotaxane network were demonstrated, including the recyclable property as the crosslinked polymer. The meaning and applicability of the reversible crosslinking/decrosslinking system developed in the polyrotaxane network are specially emphasized for unique and potential application. [DOI 10.1295/polymj.38.1]

KEY WORDS Rotaxane / Polyrotaxane / Polyrotaxane Network / Gel / Interlocked Polymer /
\end{abstract}

\section{STRUCTURE OF ROTAXANE, OLIGOROTAXANE, AND POLYROTAXANE}

Rotaxane is one of the typical interlocked molecules in addition to catenane and knot, ${ }^{1-4}$ and is characterized by high freedom and mobility of their components that are linked mechanically, as predicted from their topologically unique structures (Figure 1). Rotaxane can be regarded as a supramolecular system from viewpoint of the co-operative effect of the components. Interestingly, the components are so strongly linked in spite of the extraordinarily high mobility that it requires covalent bond energy to break the mechanical linkage or to separate the components. Therefore, the so-called "topological bond" between the components is "soft but strong bond" in comparison with the typical covalent bond and bonding in supramolecular systems. The interlocked molecules having such characteristic features are expected to have special or extraordinary physical and chemical properties. [2]Rotaxane is the most primitive level compound among rotaxanes, while [5]rotaxane is the highest level compound as the structure-discrete oligorotaxane. The chemistry of rotaxanes has well progressed in accordance with the interest in their unique structures and the expectation to development as the parts of molecular machines or molecular device, whereas that of polymers comprising of these structures as the key repeating units, i.e. interlocked polymers or polyrotaxanes, has been in a way of progress. The chemistry of [2] rotaxanes as well as [2]catenanes has recently stressed their applications by utilizing the vast amount of the fundamental studies as their background, while both synthesis and application of the interlocked polymers have studied simultaneously.

General structures of the representative polyrotaxanes are shown in Figure 2. Polyrotaxanes can be categorized into two types: one is the polyrotaxanes consisting of the main chains of covalent type as shown in the top four, while the other involves the polyrotaxanes of which monomer linking units are constructed by the rotaxane structure as shown in the following two structures. The essential difference in main chain structure between the two types of polyrotaxanes should cause large difference in physical or mechanical properties of them. The former polyrotaxanes are further divided into main chain-types and side chain-types. Poly[2]rotaxane and poly[3]rotaxane seem to be genuine polyrotaxane as "topological polyrotaxanes", which may reflect their truly unique structures to their properties.

Although characteristic properties in mechanical and/or rheological aspects have been suggested to the interlocked polymers in bulk state, the ring size of the wheel component included seems to exert a

${ }^{\dagger}$ To whom correspondence should be addressed (E-mail: ttakata@polymer.titech.ac.jp). 


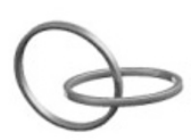

[2]Catenane

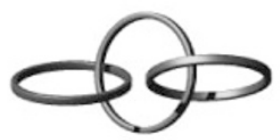

[3]Catenane

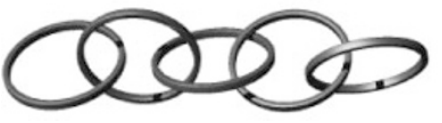

[5]Catenane

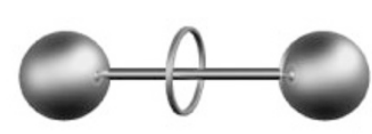

[2]Rotaxane

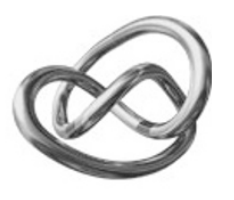

3 , knot

Figure 1. Structures of catenane, rotaxane, and knot.

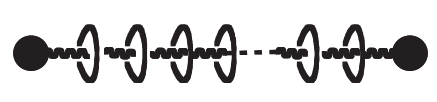

Main Chain-Type Polyrotaxane

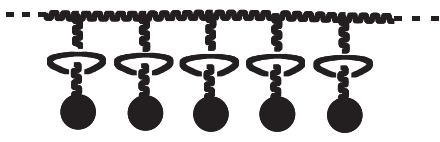

Side Chain-Type Polyrotaxane

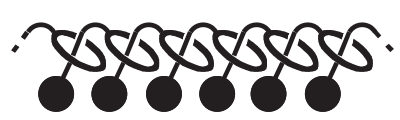

Poly[2]rotaxane

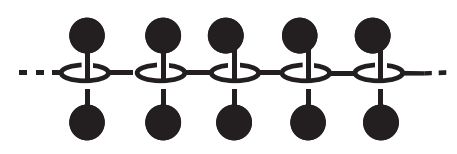

Main Chain-Type Polyrotaxane

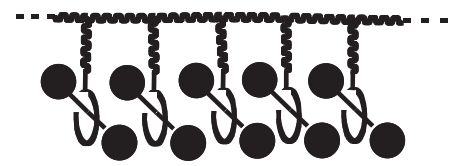

Side Chain-Type Polyrotaxane

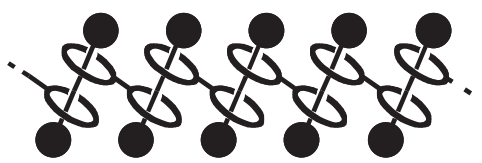

Poly[3]rotaxane

Figure 2. Structures of various polyrotaxanes.

serious influence, as well as that by the freedom of the component. As a way of looking at the polymer properties, effect of entangled polymer chain plays an important role. That is, there can be considered an elastic property based on the interlocked polymer chains as highly interpenetrated ones which may be associated with the properties of rubber and interpenetrating polymers, when a big wheel is used. Meanwhile, when a wheel component is connected with the chain polymers, bonding between the wheel component and the polymer chain results in producing the crosslinked points that can move on the chain. Such type of "topological crosslinking" can provide special mobility to the polymer, because it is distinguished from both physical and chemical crosslinkings with little mobility. As mentioned above, studies from view point of material science and technology of these new type of polymers are progressing, particularly since 21 st century has started, and various unique properties are expected for the polymers characterized by the interlocked structures.

There are many reviews including comprehensive articles of Gibson's, ${ }^{5}$ Stoddart's, ${ }^{6}$ Harada's, ${ }^{7}$ and Takata's ${ }^{8}$ on the polyrotaxanes and polycatenanes, in addition to a lot of review articles and books on the rotaxanes and catenanes, which are cited in the references. ${ }^{5-8}$

Here this review emphasizes the meaning of the concept "dynamic covalent bond chemistry" for syntheses of rotaxanes and polyrotaxanes. One purpose of the dynamic covalent bond chemistry is to effectively utilize equilibria of covalent bond compounds for molecular architecture, and thereby is actually important in the fields of supramolecules and supramolecular polymers characterized by molecular integration as the key technology for their constructions. In this review, we would like to mainly discuss the syntheses of poly[3]rotaxane and its polymer network consisting 
a. Covalent Bonding

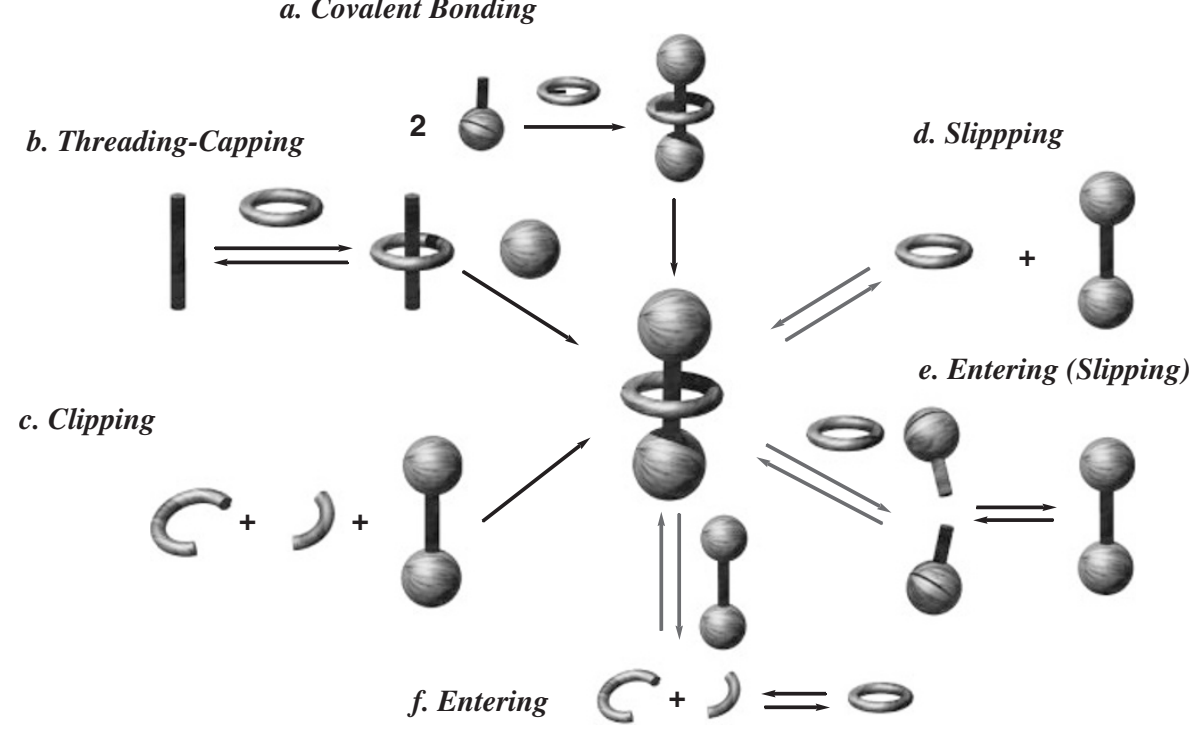

Figure 3. Typical synthetic methods of rotaxanes.

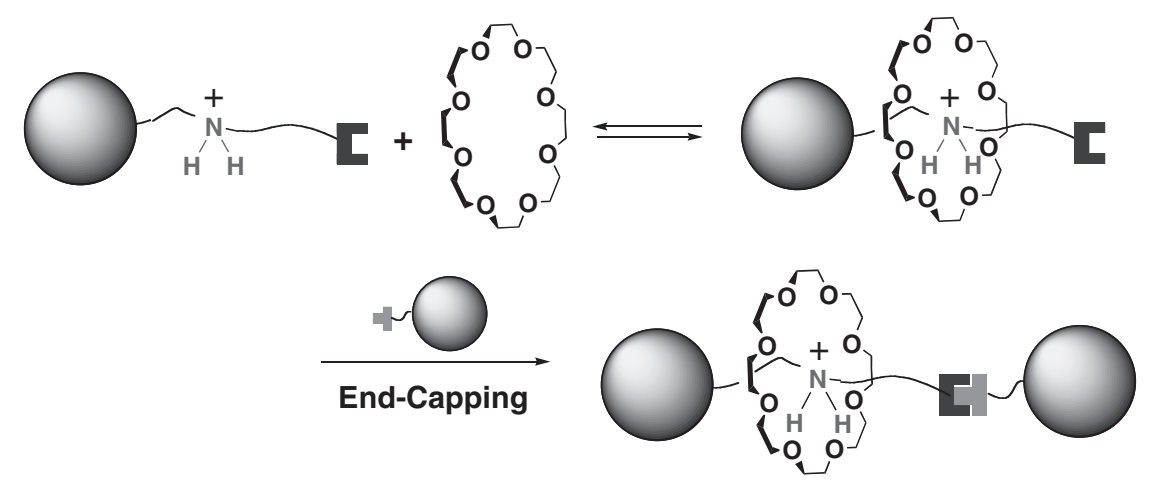

Scheme 1.

of a crown ether-sec-ammonium slat couple in our recent works, after surveying the synthesis of rotaxanes briefly, especially from viewpoint of the dynamic covalent bond chemistry.

\section{SYNTHESIS OF ROTAXANE}

Figure 3 summarizes the representative synthetic methods of [2]rotaxanes. ${ }^{3,4,8}$ Methods a-c are characterized by the kinetically controlled process as the final step to the rotaxane. In particular, method $\mathbf{b}$ is the general and most straightforward synthesis: i.e., end-capping of the axle terminal with a bulky group after threading of the axle into the wheel. Most polyrotaxanes are prepared according to this methodology. On the other hand, the methods $\mathbf{d}-\mathbf{f}$ undergo the thermodynamically controlled process as the equilibrium process in the last step (equilibrium arrows). Since the procedure in the two synthetic methods completely differs from each other, the control of the synthetic reaction is also different. The thermodynamic control process has recently attracted much attention from viewpoint of advantages in not only yield but also reaction condition, in accordance with the progress in supramolecular chemistry, since the molecular assembly approach is favored in constructions of complicated molecules.

Hydrogen Bonding Interaction Used for Complexation to Rotaxane Structure

The attractive interaction between a crown ether and a certain sec-ammonium salt is strong enough to prepare various interlocked compounds. The combination of 24-membered crown ether such as dibenzo24-crown-8-ether (DB24C8) and bis(primary alkyl) or dibenzyl ammonium salt has been widely used for the complex formation (Scheme 1). ${ }^{9,10}$ While there are two types of complexes, side-on and inclusion complexes, are possible, inclusion complex, which has pseudorotaxane structure, is generally observed unless the ammonium salt has the bulky end-caps. The rotaxane structure can be eventually fixed by end-capping. It is noteworthy that basic reaction condition should be avoided during the end-capping because the hydro- 


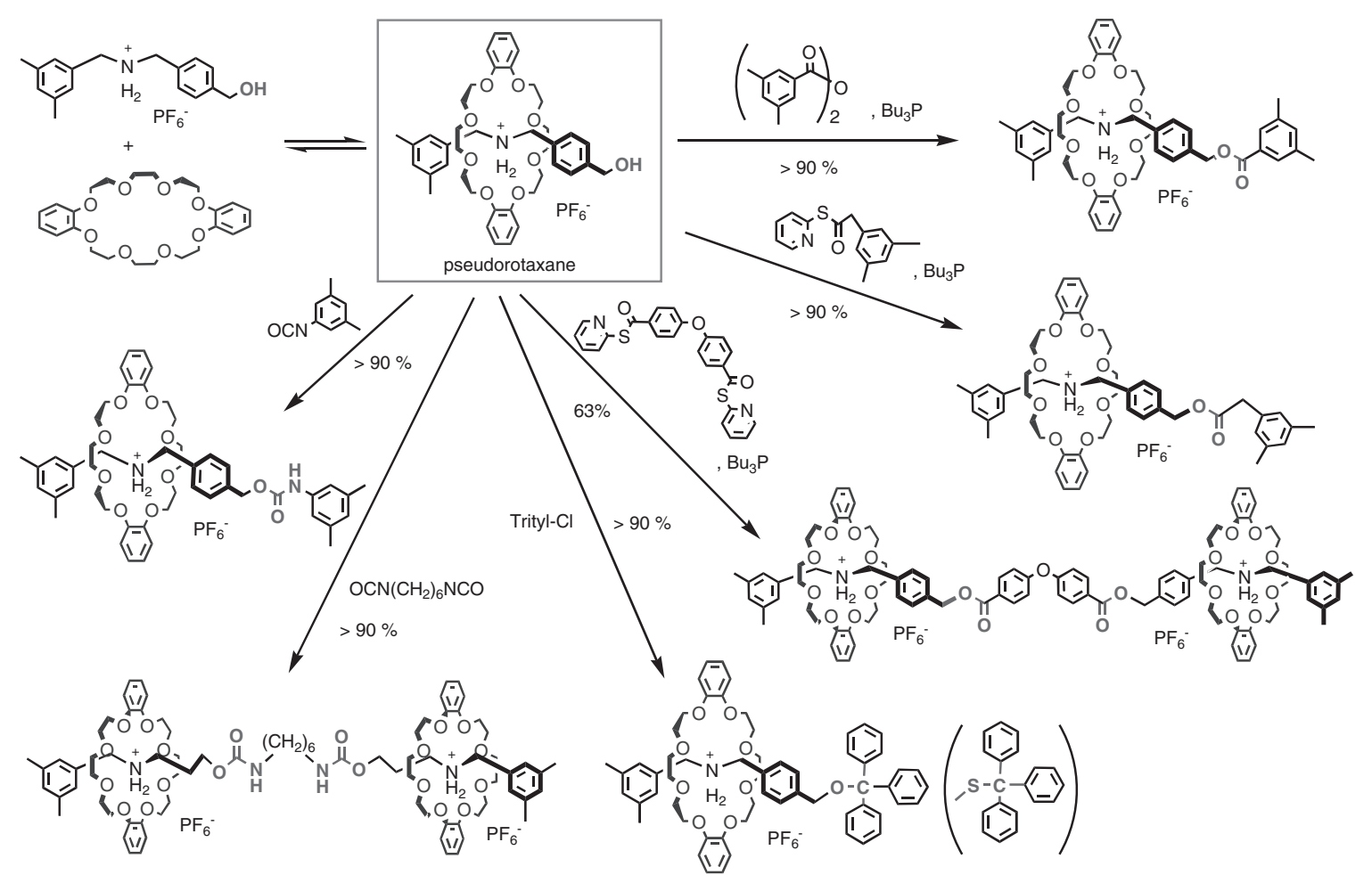

Scheme 2.

gen-bonding interaction is lost when the ammonium salt is neutralized.

Takata et al. have adopted a couple of crown ether wheel and sec-ammonium salt axle as the rotaxane components, mainly because of the synthetic versatility, i.e. a variety of structures designable for the wheels and axles and the easy construction of rotaxanes. $^{3,4}$ The synthetic methods of rotaxanes which they have developed in recent several years are summarized in Schemes 2-6.

By the elaborated studies on the syntheses of rotaxanes according to the method b, Takata et al. developed a high yielding process, i.e. acylative endcapping of hydroxy group of the axle component. ${ }^{11}$ While acylation of alcohol is usually carried out in the presence of tertially amine such as triethylamine, the use of amine significantly decreased the yield of the rotaxane, as mentioned above. Takata et al. have demonstrated that the use of the combination of tributylphosphine as a catalyst and acid anhydride as an acylation reagent is an excellent solution for this problem. ${ }^{11}$ Scheme 2 summarizes the rotaxane syntheses via acylation of axle terminal hydroxy group with acid anhydride, active ester, and isocyanate. Trityl chloride is also used for the end-capping of the terminal hydroxyl group.

The homocoupling of [2]pseudorotaxane is one of the most effective methods to prepare [3]rotaxane. Original acylative end-capping method is not effective for this type of oligorotaxane synthesis, however, because of difficulty in obtaining bifunctional acid anhydride. This restriction was solved by the use of active ester instead of acid anhydride. Actually Takata et al. prepared [3] rotaxane in a good yield by the active ester method. ${ }^{12}$ The more effective method for the homocoupling of [2]pseudorotaxane is achieved by the oxidation of mercapto group to form disulfide. The high yielding synthesis of [3] rotaxane was accomplished by Busch et al. by the oxidative coupling of [2]pseudorotaxane bearing the mercapto group at the terminus by iodine. ${ }^{13}$

The acid-catalyzed acylation of both terminal hydroxyl groups can be done with acid anhydride and acid chloride-silver triflate (Scheme 3). ${ }^{14}$ The method is well applicable to the synthesis of oligo and polyrotaxanes, as shown below.

Synthesis of Oligo and Polyrotaxanes by ThreadingCapping Protocol Using Crown Ether-sec-Ammonium Couple

Various reaction conditions have been proposed to construct oligo- and polyrotaxanes without the use of base. The first preparation of oligorotaxane consisting of crown ether and ammonium salt was carried out by Stoddart et al. via the 1,3-dipolar cycloaddition of azide to di-tert-butyl acetylenedicarboxylate. ${ }^{15,16}$ Although the 1,3-dipolar cycloaddition took place under neutral condition, the yield of the rotaxane was rather low because the higher reaction temperature which suppressed the pseudorotaxane formation was necessary.

Takata et al. found that oligorotaxanes were easily 


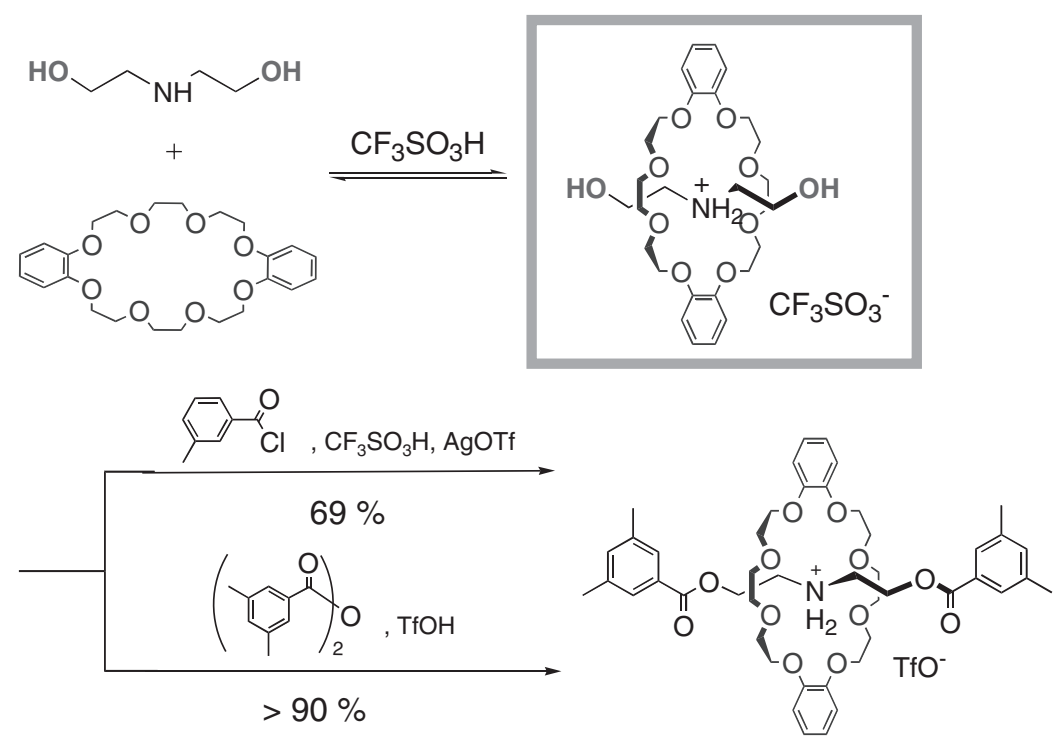

Scheme 3.
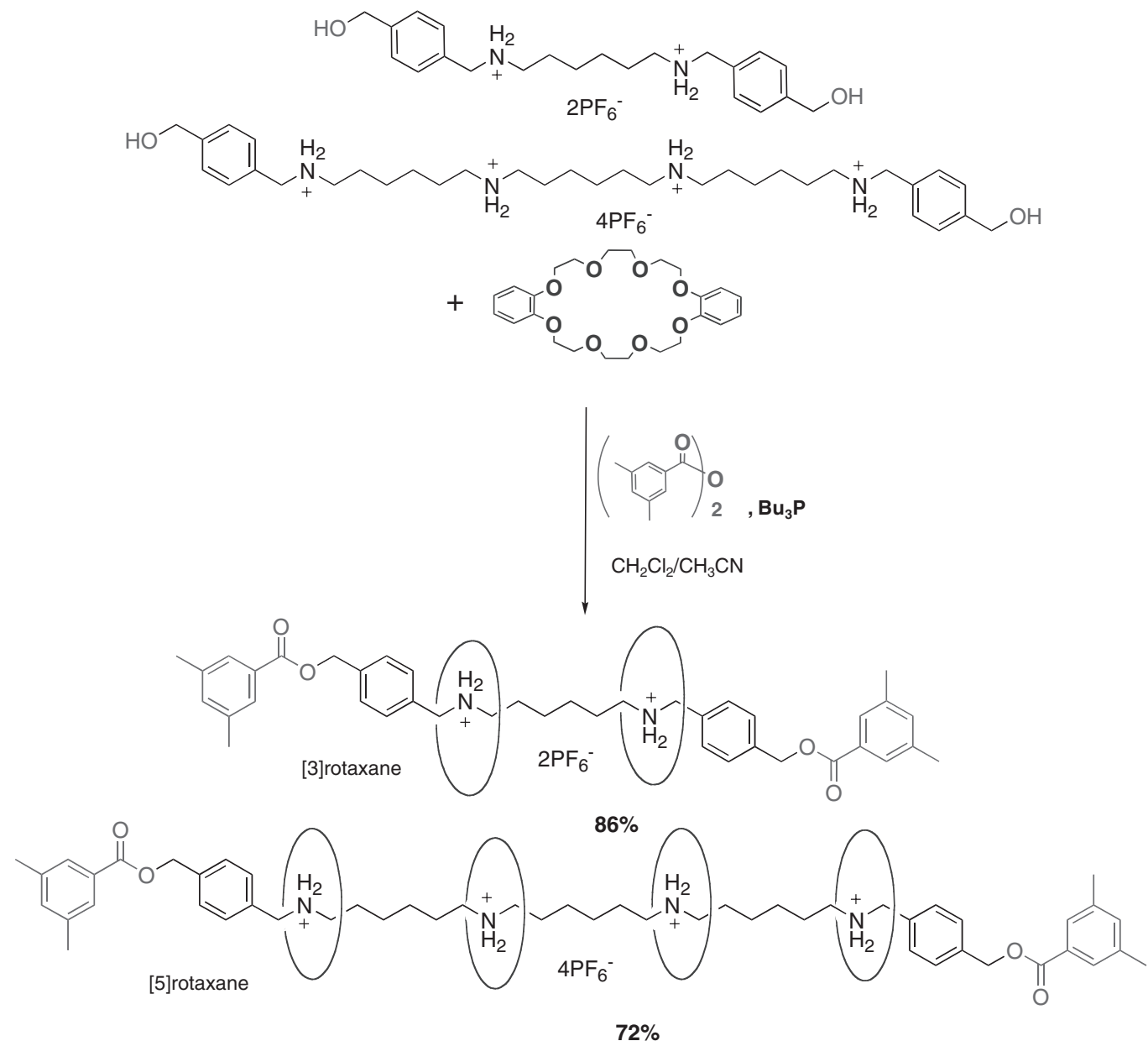

Scheme 4.

prepared in more than $70 \%$ yield by phosphine-catalyzed acylative end-capping method ${ }^{17}$ (Scheme 4). Table I shows the effect of spacer length of the axle component on the yield of [3] rotaxane. The result suggested that the 6 methylene distances between the neighboring ammonium groups are needed to suppress the interaction between two wheels, when DB24C8 is used.

Synthesis of polyrotaxane by a similar acylative end-capping reaction was carried out using nylon- 
based polyamine possessing benzylic hydroxyl groups at both termini (Scheme 5). Complete formation of pseudopolyrotaxane by treating with triflic acid in the presence of excess DB24C8 was confirmed. Successive end-capping of the hydroxyl groups of the pseudopolyrotaxane with 3,5-dimethylbenzoic anhy-

Table I. Effect of spacer length on the yield of [3] rotaxane in end-capping synthesis

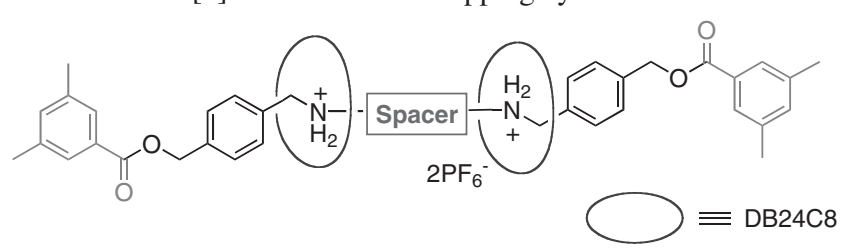

\begin{tabular}{ccc}
\hline \multirow{2}{*}{$\begin{array}{c}\text { ammonium salt } \\
\text { spacer }\end{array}$} & \multicolumn{2}{c}{ yield (\%) } \\
\cline { 2 - 3 } [2]rotaxane & [3]rotaxane \\
\hline$\left(\mathrm{CH}_{2}\right)_{2}$ & 23 & 0 \\
$\left(\mathrm{CH}_{2}\right)_{4}$ & 30 & 51 \\
$\left(\mathrm{CH}_{2}\right)_{6}$ & 36 & 64 \\
$-\mathrm{CH}_{2}-\mathrm{CH}_{2}-$ & 64 \\
\hline
\end{tabular}

dride yielded $80 \%$ rotaxanated polyrotaxane in a good yield.

In addition to the above main chain-type polyrotaxane, two side chain-type polyrotaxanes were also prepared by radical polymerization of vinylic pseudorotaxane monomers which contained either of couples of crown ether wheel-sec ammonium axle and paraquate type wheel and oxyethylene axle (Figure 4). ${ }^{18-20}$

\section{DYNAMIC COVALENT BOND CHEMISTRY IN ROTAXANE SYNTHESIS}

A variety of rotaxanes is prepared by utilizing dynamic covalent bond. ${ }^{20}$ Since disulfide bonding is labile, and disulfide-exchange reaction is catalyzed by the certain nucleophile such as thiol, a few wonderful methods to prepare rotaxanes based on the dynamic covalent chemistry are developed by Takata et al. ${ }^{21-23}$ When a bifunctional secondary ammonium salt bearing disulfide linkage and bulky end-caps was mixed with dibenzo-24-crown- 8 and a catalytic amount of benzenethiol, crown ether entered into the
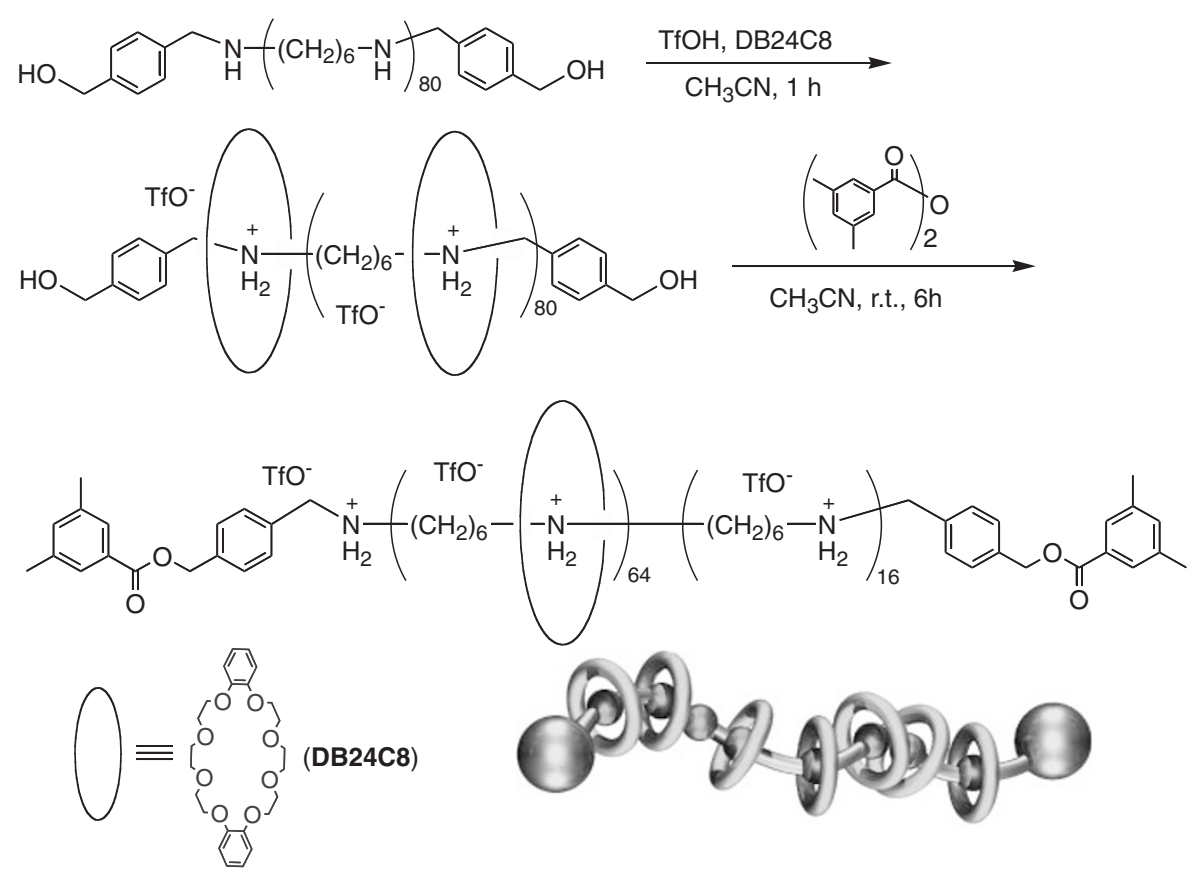

Scheme 5.
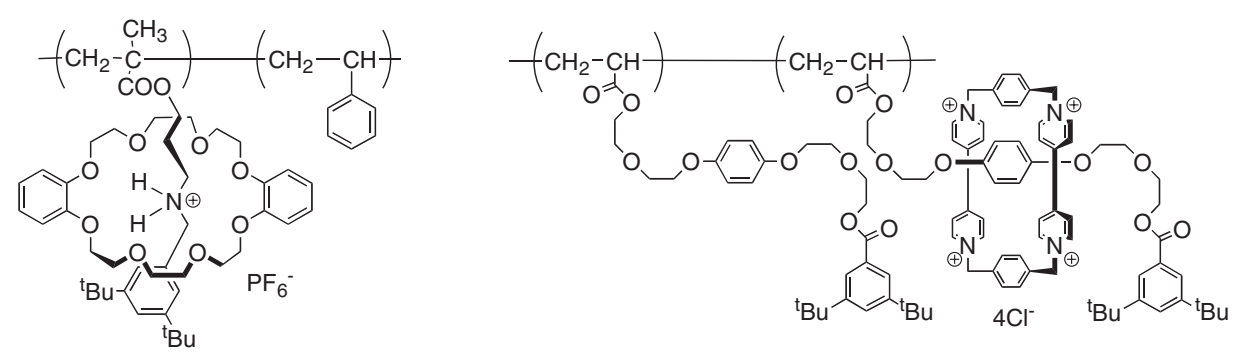

Figure 4. Structures of side chain-type polyrotaxanes. 

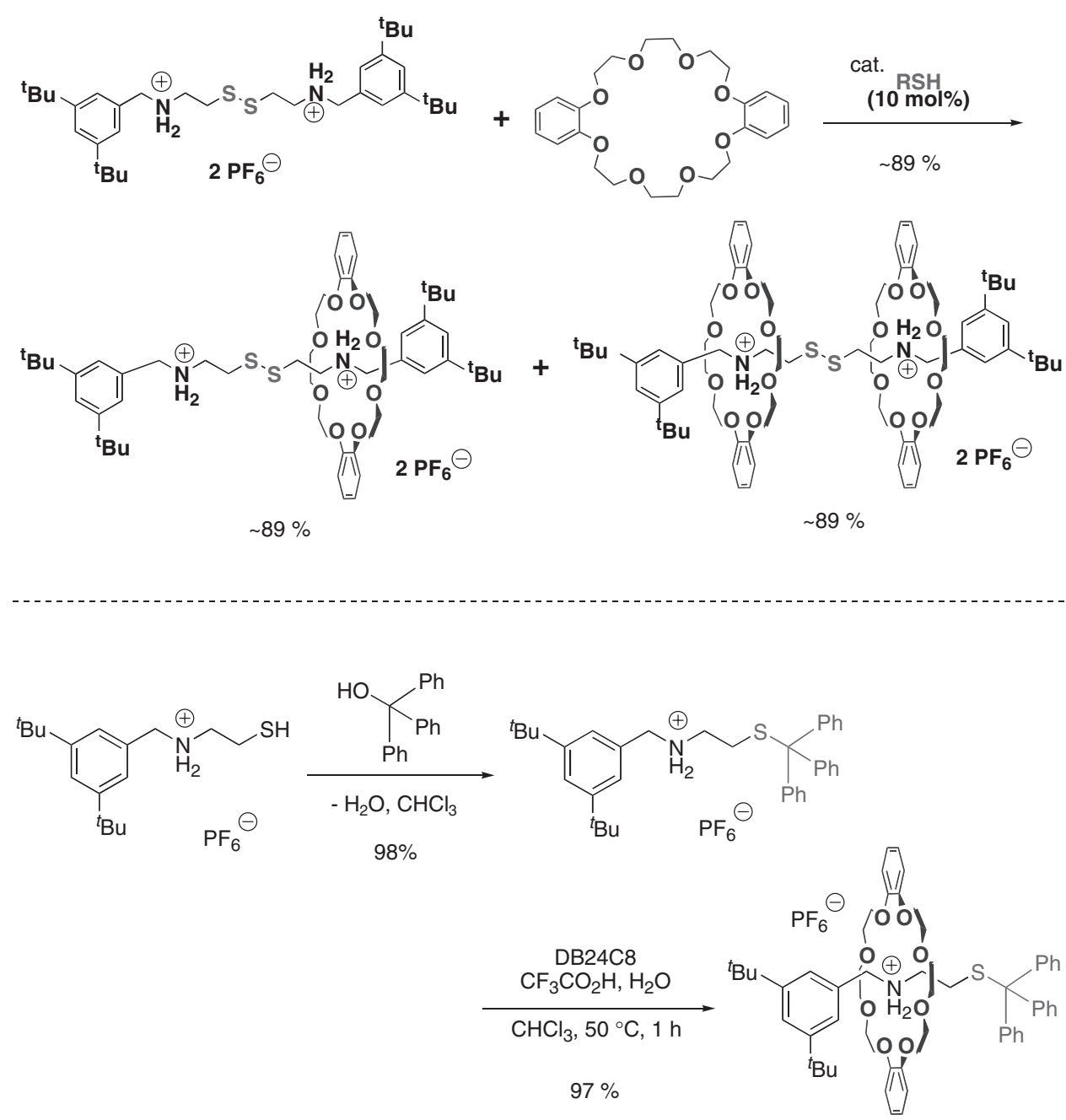

Scheme 6.

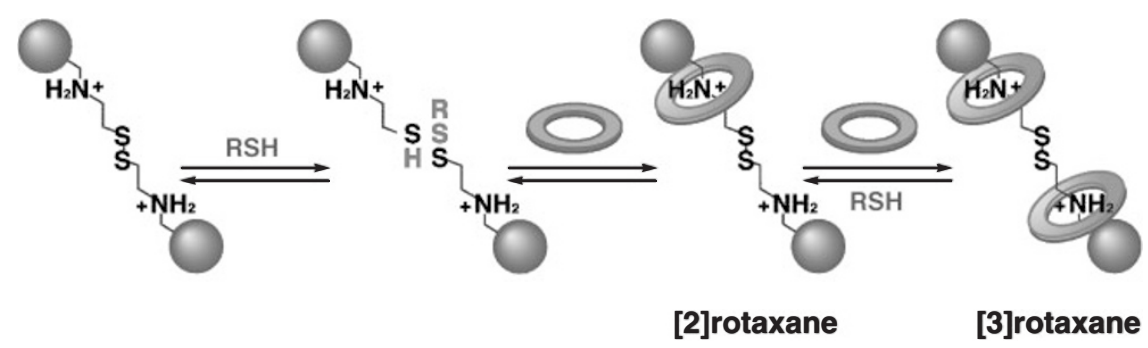

Scheme 7.

disulfide linkage to afford [2] and [3] rotaxanes ${ }^{21,22}$ (Scheme 6). The mechanism of the formation of these rotaxanes is simply illustrated in Scheme 7, which is so called "unlock-lock" process. The initial SS bond cleavage by the catalysis of thiol results in the formation of half rotaxane or pseudorotaxane both of which can thread in the wheel and undergo end-capping as "unlock" process to give [2]rotaxane. Similar process gives [3] rotaxane. These two rotaxanes were selectively produced in more than $80 \%$ yield by choosing the suitable conditions. Since all processes are under equilibria, yield of the rotaxanes is easily controlled by temperature, concentration, polarity of solvent and so on.

Trityl sulfide $\mathrm{C}-\mathrm{S}$ bond is also labile enough to be regarded as dynamic covalent bond. As depicted in Scheme 6, a mixture of DB24C8 wheel and an axle component having central sec-ammonium group and terminal trityl sulfide group is treated with trifluoroacetic acid in the presence of trace amount of water to afford corresponding [2] rotaxane in $\sim 97 \%$ yield. $^{23}$ Reversible $\mathrm{C}-\mathrm{S}$ bond cleavage gives rise to the high yielding preparation of rotaxane. 
Covalently-linked polymer backbone

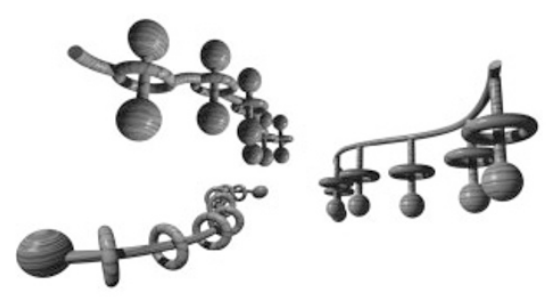

main chain-type

side chain-type
Topologically-linked polymer backbone

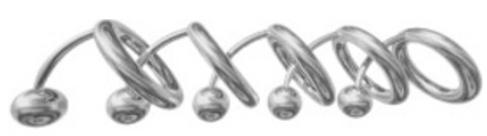

poly[2]rotaxane

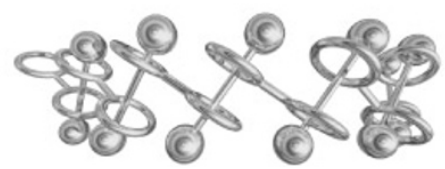

poly[3]rotaxane

Figure 5. Schematically illustrated polyrotaxanes.

\section{SYNTHESIS OF POLY[3]ROTAXANES}

Synthesis of polyrotaxanes is performed basically by using or applying the synthetic methods of rotaxanes as shown in Figure 3. A large number of main chain-type polyrotaxanes have been synthesized to date, together with several side chain-type ones (Figures 2 and 5). ${ }^{5-8}$ Meanwhile, there are few reports on polyrotaxanes having backbones consisting of mechanical bonds as well as poly[2]catenanes. ${ }^{5-8,24}$ Polyrotaxanes, especially those consisting of "topologically linked polymer backbones" are quite fascinating, because their main chains involve rotaxane's mechanical bonds directly linking the repeating units, in contrast to those of the main chain-type polyrotaxanes, i.e. poly[2]rotaxane and poly[3]rotaxane (Figure 5). Several groups have recently reported attempts to synthesize poly[2]rotaxanes (daisy chains), obtaining cyclic dimers or trimers instead of the polymers. ${ }^{25-29}$ Thus, the synthesis of this type of polyrotaxanes is not only challenging but also promising for creating new polymer materials with unique physical properties.

\section{Synthesis of Polypseudo[3]rotaxane}

Poly[3]rotaxane has long been a challenging target and the synthetic strategy of poly[3]rotaxane or polypseudo[3]rotaxane is illustrated by Scheme 8, which consists of self-assembly of two homoditopic monomers such as dumbbell and glass-shaped molecules. ${ }^{30}$

In early 1999, Gibson's group demonstrated how it could be realized in the system of pseudopolyrotaxane (Scheme 9). ${ }^{31}$ They showed that bis(24-crown-8 ether) and bis(secondary ammonium salt) formed cyclic dimer predominantly at low concentrations below 0.010 $\mathrm{M}$ and resulted in the formation of linear polypseudo[3] rotaxane at high concentrations above $1.0 \mathrm{M}$. They prepared flexible, amorphous, and transparent films by casting from 1:1 stoichiometric mixture of the two monomers. Thus, the formation cyclic dimers can be suppressed by increasing feed concentrations.

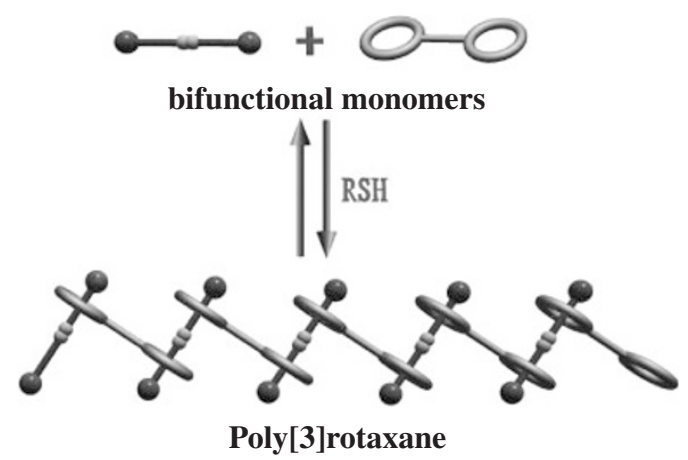

Scheme 8.

Two years later, Takata and his co-workers reported a new protocol for the synthesis of polypseudorotaxanes (Scheme 10). ${ }^{32}$ Model reaction using cyclohexyl-terminated bifunctional sec-ammonium salt and DB24C8 revealed the high yielding formation of doubly complexed product, i.e. the possibility of occurrence of polymerization. They employed homoditopic molecules, that is, bis(dibenzo-24-crown-8 ether) and bis(secondary ammonium salt) having two cyclohexyl groups at both ends, as monomers. They conducted "polyslipping", namely, continuous slipping process which relies on the size-complementarity between cyclohexyl group and dibenzo-24-crown-8 ether. The polyrotaxane-like supramolecule (polypseudo[3]rotaxane) thus obtained behaves like a polyrotaxane under appropriate conditions, i.e., the polyrotaxane is kinetically stable at ambient temperature in less polar solvent. Upon immersion in a polar solvent such as DMSO at room temperature, however, the polyrotaxane was quantitatively disassembled into the monomers in a short time. By definition, the polypseudo[3]rotaxane is not a polyrotaxane but a polypseudorotaxane, since the end-capping groups are not bulky enough to prevent deslipping under all circumstances. Anyhow, the polypseudo[3]rotaxane has a more rotaxane-like character than other polypseudorotaxanes reported to date and can be categorized in a new class of polymeric compounds. 

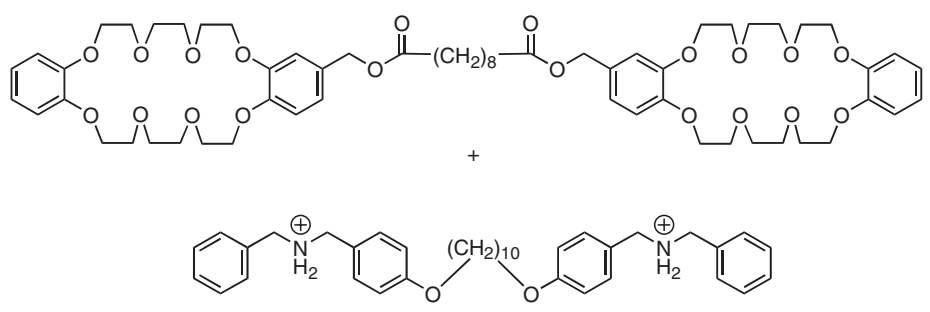

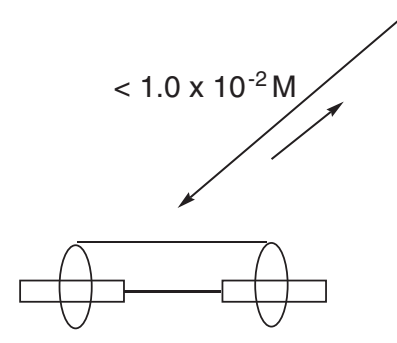

cyclic pseudorotaxane

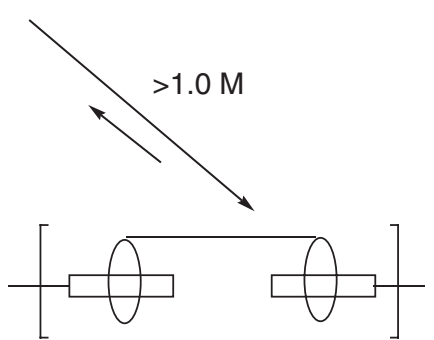

pseudopoly[3]rotaxane

Scheme 9.
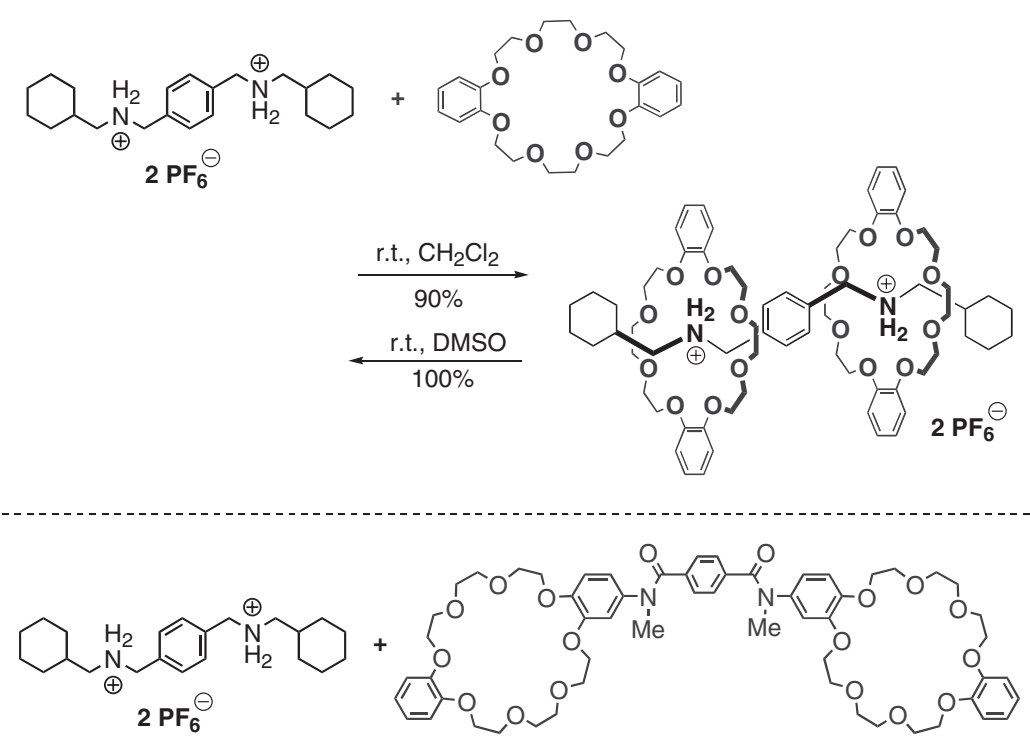

rt in DMSO $\uparrow \downarrow \Delta$ in $\mathrm{CD}_{3} \mathrm{CN}$

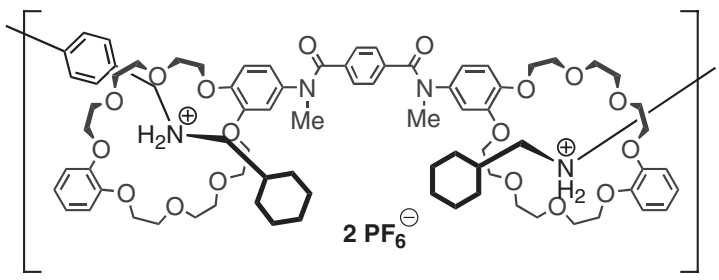

Polypseudo[3]rotaxane

Scheme 10.

Synthesis of Poly[3]rotaxane According to the Concept of Dynamic Covalent Bond Chemistry

The synthesis of first poly[3]rotaxane, a real tour de force, was finally accomplished by Takata's group in early 2003. ${ }^{30}$ As mentioned above (Scheme 6), Takata et al. have reported a new synthetic method of [2]- and [3]rotaxanes under thermodynamic control, which utilizes the reversible cleavage-rebondage process of the disulfide bond in the presence of a catalytic amount of thiol. ${ }^{33}$ The yields of the rotaxanes can be well-controlled by the solvent, concentration, and temperature, since the whole process is reversible. 
Important features of this method are the efficient thiol-disulfide interchange reaction under mild conditions and the tolerance of disulfide for many functional groups. ${ }^{33}$ Therefore, this method is promising for the synthesis of more complex molecules such as polyrotaxanes.

Prior to the polymerization, degree of polymerization between the components in Scheme 9 was estimated from the complexation ratio between bis(ammonium salt) having a centrally located disulfide linkage and DB24C8 (Scheme 11). A 1:2 mixture of them was treated with benzene thiol $(4 \mathrm{~mol} \%)$ in $\mathrm{CHCl}_{3} / \mathrm{CH}_{3} \mathrm{CN}$ to give a mixture of [2] rotaxane and [3]rotaxane in 10 and $89 \%$ yields, respectively. The yields clearly indicated the degree of polymerization of $c a .30\left(M_{\mathrm{n}} c a .30000\right)$, when the bifunctional crown

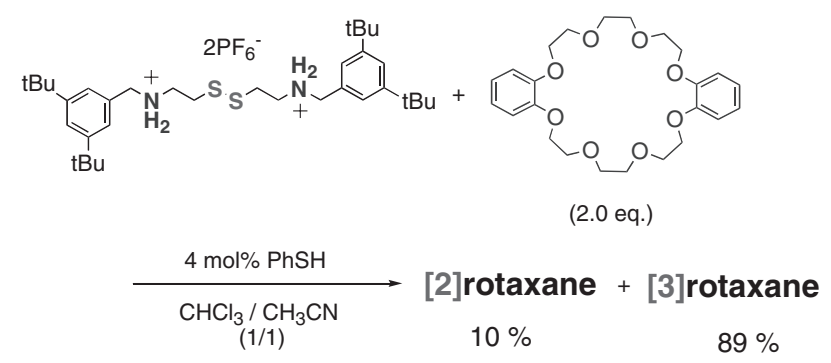

Scheme 11. ether is employed.

A homoditopic monomer, bis(dibenzo-24-crown-8 ether), was synthesized from dibenzo-24-crown-8 ether in four steps,${ }^{32}$ whereas bis-ammonium salt having a disulfide bond at the center was prepared according to the reported method (Scheme 12). ${ }^{21}$

When the polymerization was carried out by allowing a mixture of $0.50 \mathrm{M}$ mixture of the monomers and a catalytic amount of benzenethiol $(0.004 \mathrm{M})$ in $\mathrm{CHCl}_{3} / \mathrm{CH}_{3} \mathrm{CN}(7 / 3)$ to stand at $50{ }^{\circ} \mathrm{C}$, the corresponding poly[3]rotaxane was formed through noncovalent step growth polymerization (Scheme 13), ${ }^{30}$ of which degree of polymerization (DP) was estimated to be 7 as monitored by ${ }^{1} \mathrm{H}$ NMR at the equilibrium state (Scheme 13). GPC of the polymer showed a bimodal distribution, as shown in Figure 6. The $M_{\mathrm{n}}$ of the mixture was estimated to be $1.6 \mathrm{~kg} \cdot \mathrm{mol}^{-1}$, which was much smaller than $6.8 \mathrm{~kg} \cdot \mathrm{mol}^{-1}$, the value expected from the ${ }^{1} \mathrm{H}$ NMR spectrum. From the lower molecular weight part (LMW) [1+1]cyclic [2]rotaxane was isolated by preparative GPC, among some possible cyclic oligomers.

From the GPC analyses of the polymerizations at various concentrations, the higher molecular weight part (HMW) increased while the LMW decreased, with an increase in concentration (Figure 6 and Table II).
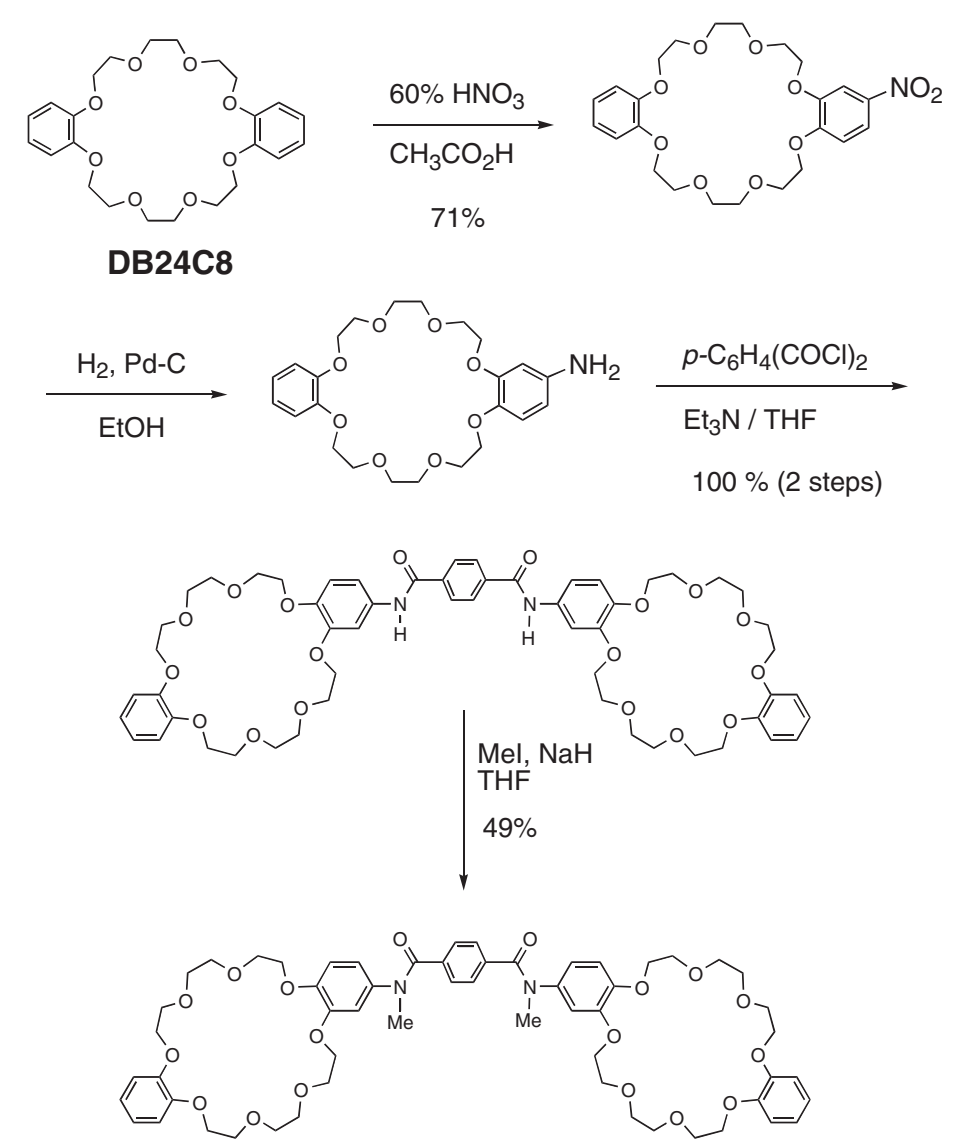

Scheme 12. 


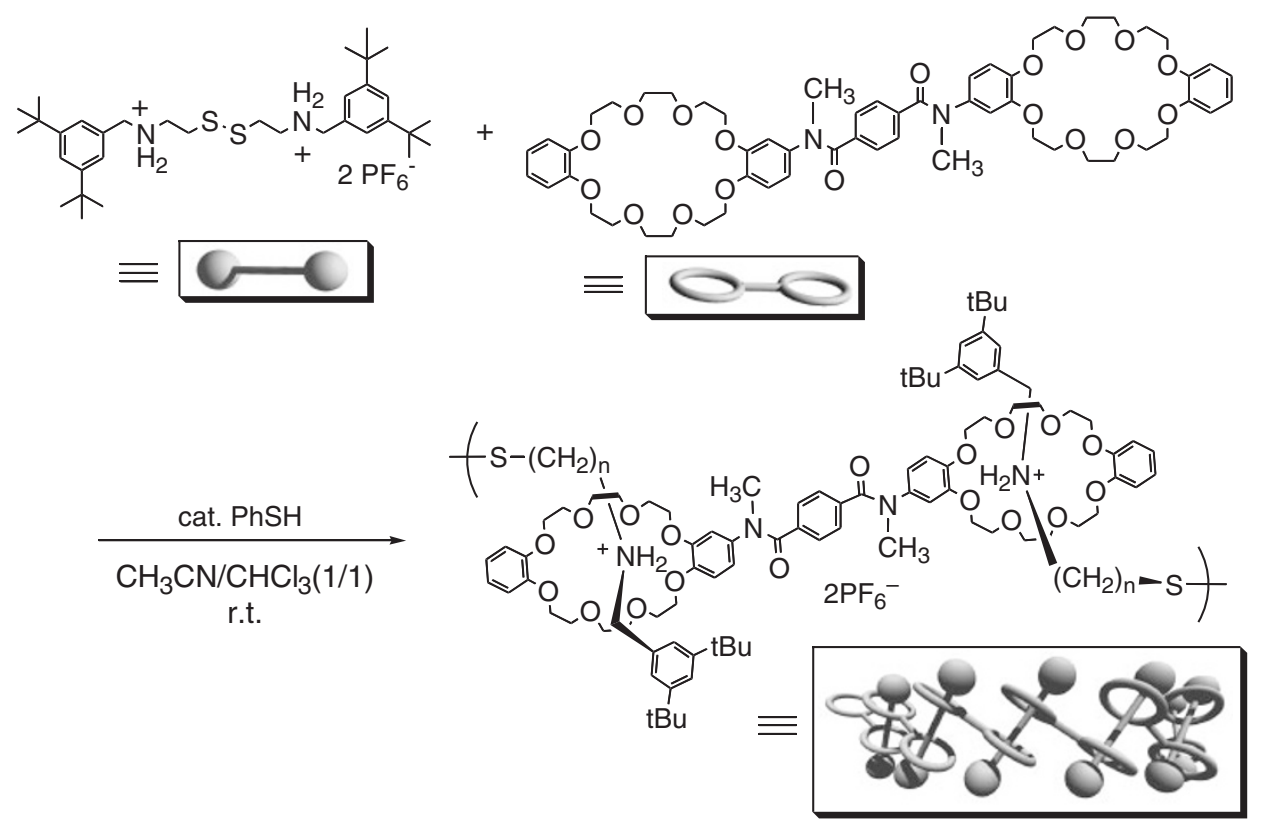

Scheme 13.

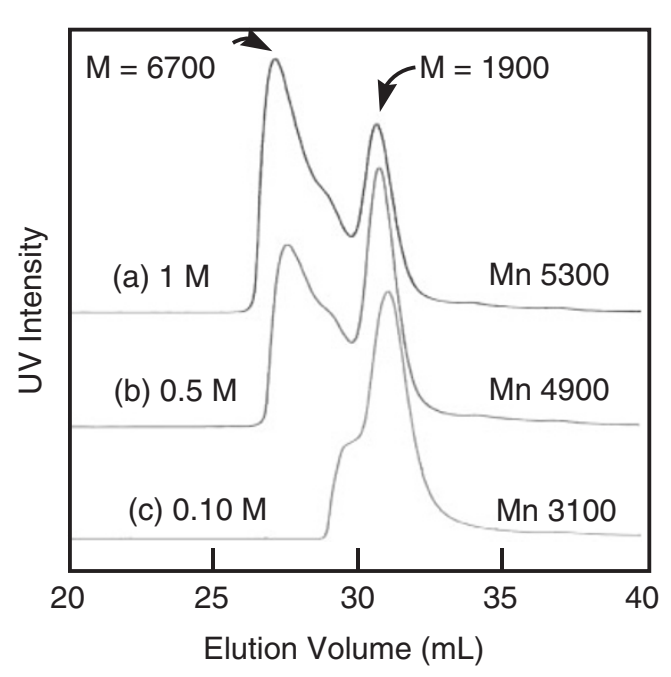

Figure 6. GPC profiles of the polymerizations at various concentrations.

To avoid the obscurity of GPC determination of polymer molecular weight coming from the use of polystyrene standards for calibration, the mechanical bonds and the ammonium salt moieties of the polymer, and so on, the DPs was determined by end-group analysis with ${ }^{1} \mathrm{H}$ NMR. The analysis was carried out by monitoring several possible tert-butyl signals of the terminal structure, central structure, and side products. Figure 7 displays the partial ${ }^{1} \mathrm{H}$ NMR spectra in the region from 1.4-1.1 ppm where all tert-butyl signals are observable. The DP of HMW polymer collected by HPLC in $64 \%$ yield was estimated to be 20-25 by ${ }^{1} \mathrm{H}$ NMR, which corresponds to a $M_{\mathrm{n}}$ of $19-23 \mathrm{~kg} \cdot \mathrm{mol}^{-1}$, while that in $35 \%$ yield was $33-50$, $M_{\mathrm{n}}$ of $31-94 \mathrm{~kg} \cdot \mathrm{mol}^{-1}$.
Table II. Number-average molecular weight $\left(M_{\mathrm{n}}\right)$ and polydispersity $\left(M_{\mathrm{w}} / M_{\mathrm{n}}\right)$

\begin{tabular}{ccccc}
\hline $\begin{array}{c}{[\mathrm{C}]} \\
\text { Monomer }^{\mathrm{a}} \\
\left(\mathrm{mol} \cdot \mathrm{L}^{-1}\right)\end{array}$ & $\begin{array}{c}{[\mathrm{C}]} \\
\mathrm{PhSH} \\
\left(\mathrm{mol} \cdot \mathrm{L}^{-1}\right)\end{array}$ & $\begin{array}{c}M_{\mathrm{n}}\left(M_{\mathrm{w}} / M_{\mathrm{n}}\right) \\
\text { of HMW } \\
\left(\mathrm{kg} \cdot \mathrm{mol}^{-1}\right)^{\mathrm{b}}\end{array}$ & $\begin{array}{c}M_{\mathrm{n}}\left(M_{\mathrm{w}} / M_{\mathrm{n}}\right) \\
\text { of LMW } \\
\left(\mathrm{kg} \cdot \mathrm{mol}^{-1}\right)^{\mathrm{b}}\end{array}$ & $\begin{array}{c}\text { Area } \\
\text { Ratio }^{\mathrm{c}}\end{array}$ \\
\hline 0.10 & 0.0040 & $3.1(1.0)$ & $1.6(1.1)$ & 0.13 \\
0.50 & 0.0040 & $4.9(1.1)$ & $1.7(1.1)$ & 0.95 \\
1.0 & 0.0040 & $5.3(1.1)$ & $1.7(1.1)$ & 1.8 \\
\hline
\end{tabular}

${ }^{\mathrm{a} A n}$ equimolar amount of comonomer was employed. ${ }^{b}$ Estimated by GPC based on polystyrene standards. ${ }^{c}$ Peak area ratio of the higher molecular weight part to the lower molecular weight part.

Thus, the first synthesis of poly[3]rotaxane was achieved via a non-covalent step growth polymerization utilizing the reversible thiol-disulfide interchange reaction. ${ }^{30}$ On treatment of poly[3]rotaxane with $\mathrm{PBu}_{3}$ and $\mathrm{H}_{2} \mathrm{O}$, the disulfide group was easily reduced to give thiol quantitatively (Scheme 14). This suggests the possibility of the poly[3]rotaxane as a new type of recyclable polymer.

\section{SYNTHESIS OF POLYROTAXANE NETWORK}

Formation of Polyrotaxane Network via Statistical Crosslinking during Polymerization

Use of mechanical bonds as crosslinking points was first reported by Gibson's group. ${ }^{34-38}$ They obtained gel products in polycondensation with a 32-membered macrocyclic diol and with a 32-membered macrocyclic dicarboxylic acid. The crosslinking through threading occurred to form three-dimensional polyrotaxane networks in the cases of polyamides, ${ }^{35}$ poly- 


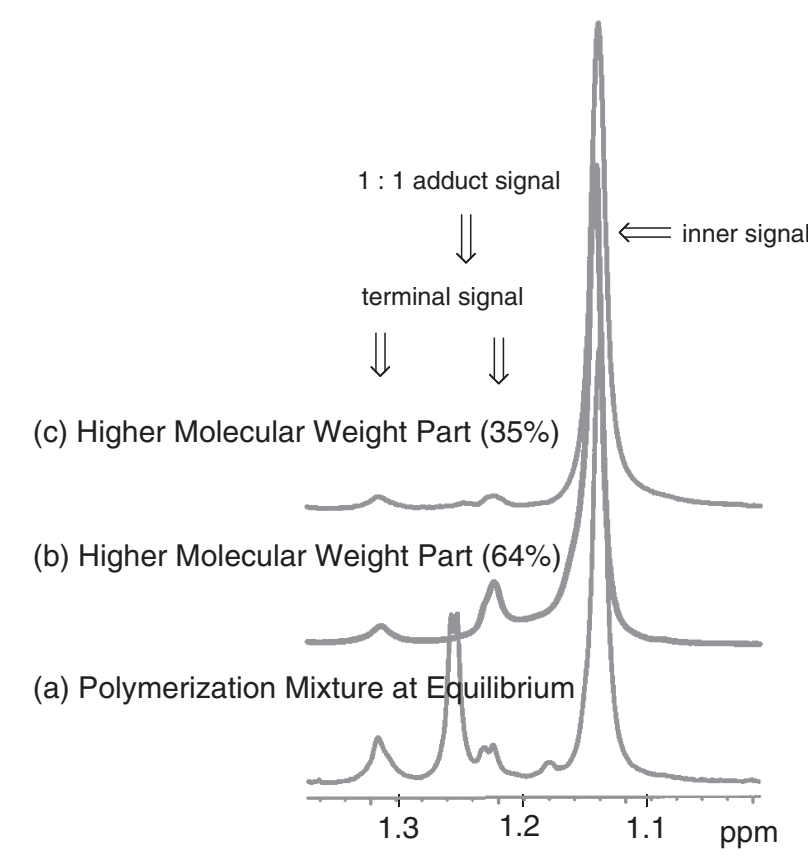

Figure 7. Partial ${ }^{1} \mathrm{H}$ NMR spectra $\left(\mathrm{CDCl}_{3}\right)$ of (a) the polymerization mixture, (b) $\mathrm{HMW}_{64}$, (c) $\mathrm{HMW}_{35}$ (the monomer feed concentration $1.0 \mathrm{~mol} \cdot \mathrm{L}^{-1}$ and $[\mathrm{PhSH}]=0.040 \mathrm{~mol} \cdot \mathrm{L}^{-1}$ in $\mathrm{CDCl}_{3} /$ $\mathrm{CD}_{3} \mathrm{CN}(1 / 1)$, r.t.).

esters, ${ }^{36}$ and polyurethanes. ${ }^{37}$ According to their first report, ${ }^{35}$ direct polycondensation of the 32-membered crown ether and an aromatic diamine to give crosslinked amide polymer, which were insoluble in any solvents (Scheme 15). This remarkably low solubility was the only one evidence, from which the rotaxane structures were deduced. Later on, Gibson et al. investigated crosslinked polyurethanes synthesized from a 32-membered macrocyclic diol and a diisocyanate in a similar manner to that employed for the crosslinked polymer by using two-dimensional ${ }^{1} \mathrm{H}$ NMR technique, and they observed some NOEs which they attributed to the presence of the rotaxane structures. ${ }^{37}$
Zilkha's group also reported a series of papers describing the synthesis of mechanically crosslinked polymer through vinyl polymerization. ${ }^{39-41}$ They employed the radical copolymerization of a $32-\mathrm{mem}-$ bered crown ether derivatives having one polymerizable double bond with styrene or methyl methacrylate (Scheme 15). Sufficient chain threading repeatedly took place to produce gelled products when the charged amount of vinyl comonomer remained to be no more than 40 equiv relative to the macrocyclic monomer. Employing a similar protocol, Tezuka's group recently prepared a gel product by the radical copolymerization of a methacrylate-functionalized cyclic poly(tetrahydrofuran) and methyl methacrylate. ${ }^{42}$

Kubo et al. employed a similar protocol to those of Zilkha's group and Tezuka's group to prepare mechanically crosslinked polymers. ${ }^{43}$ The copolymerization of a styrene-functionalized cyclic polystyrene with tert-butyl acrylate in benzene at $70^{\circ} \mathrm{C}$ yielded an insoluble polymer with a good swelling property. The insoluble material was converted to soluble polymers by cleavage of the macrocyclic units from the backbone chain of poly(styrene-co-tert-butyl acrylate). Kubo et al. attributed these results to its mechanical crosslinks formed by the threading of the polymer main chain through the macrocyclic unit during the copolymerization.

Ito and his co-worker first demonstrated in 2001 how nicely mechanical linkages worked as crosslinks of gel materials (Scheme 16). ${ }^{44,45}$ They prepared a polyrotaxane consisting of $\alpha$-cyclodextrin poly(ethylene glycol) according to the well-established procedure of Harada's group. In this case, PEG bisamine with a molecular weight of 20000 was used as the axle. The polyrotaxane had a sparse population of $\alpha$ cyclodextrin, i.e., the PEG units were "naked". Crosslinking was carried out by the reaction with cyanuric
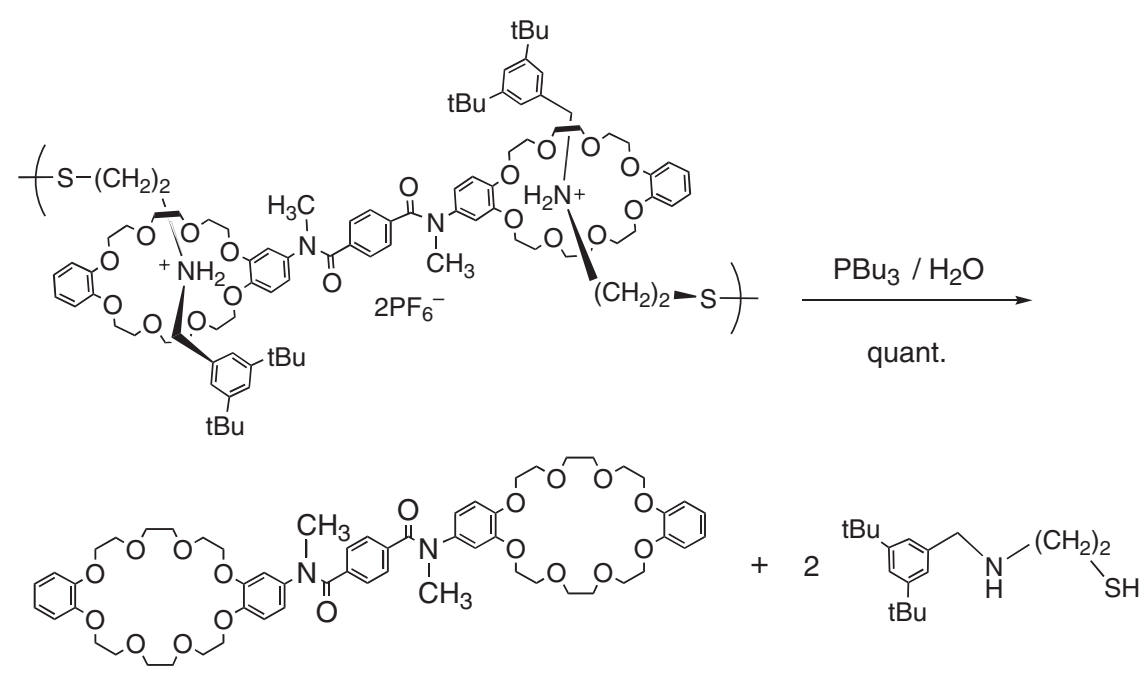

Scheme 14. 
<smiles>Nc1ccc(Oc2ccc(N)cc2)cc1</smiles>

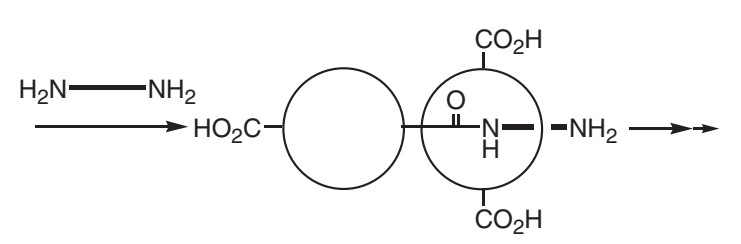

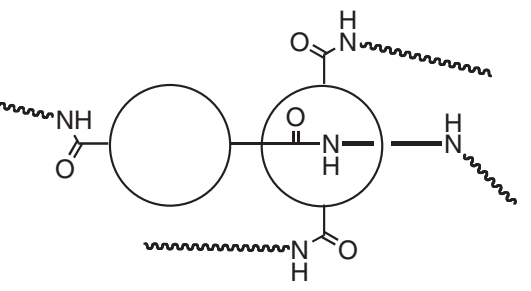<smiles>C=Cc1ccccc1</smiles>

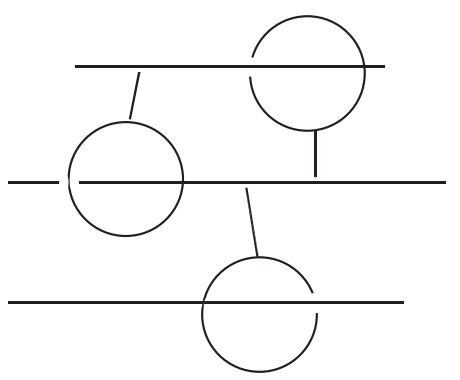

Scheme 15.
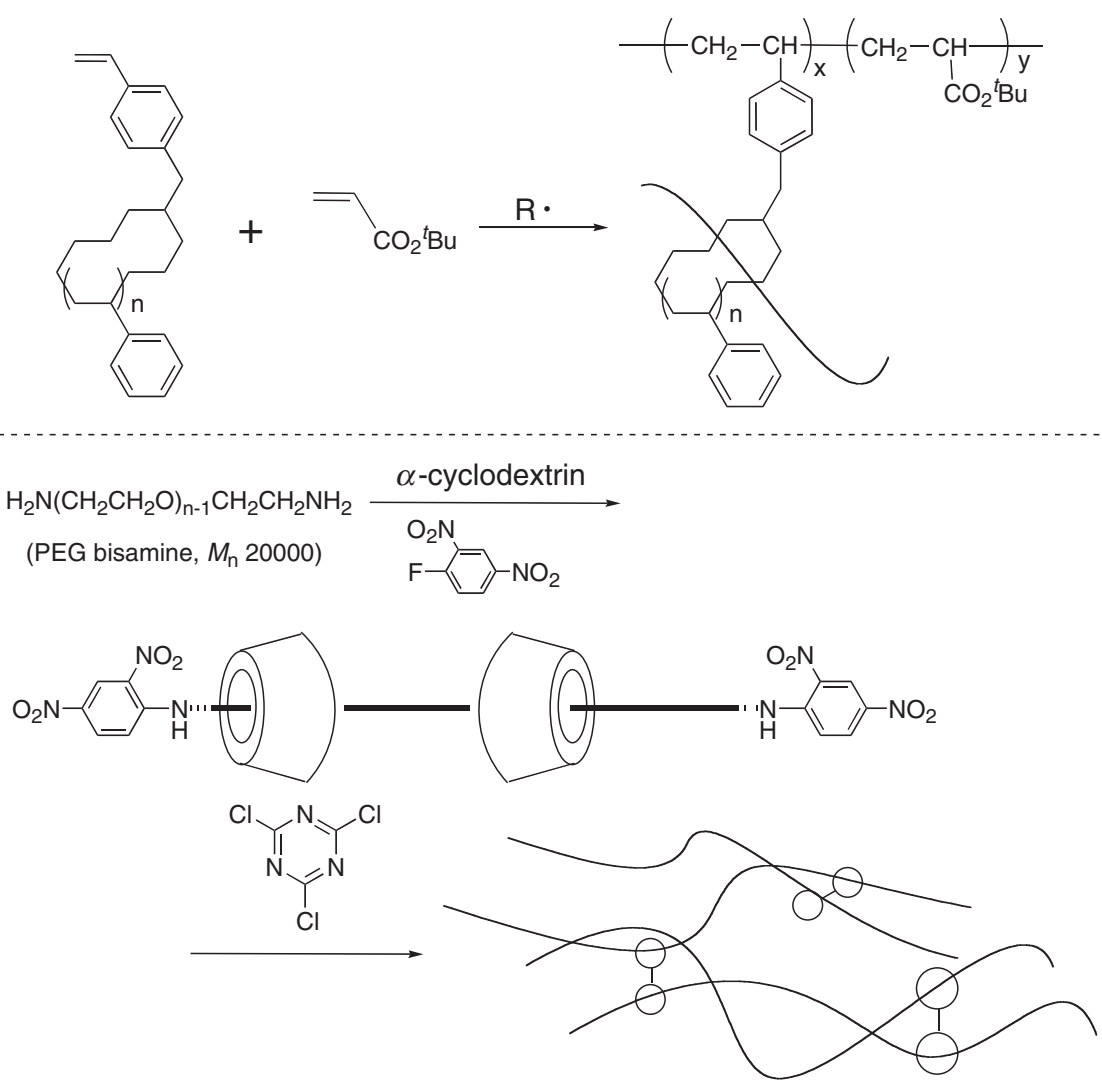

Scheme 16.

chloride to yield the crosslinked polymer as a transparent gel. The gel polymer is super absorbent and can take up water up to $c a .400$ times the dry weight. ${ }^{44}$
Yui's group has been developing the design of biodegradable hydrogels crosslinked by topological bonds. ${ }^{46-48}$ They employed polyrotaxanes consisting 


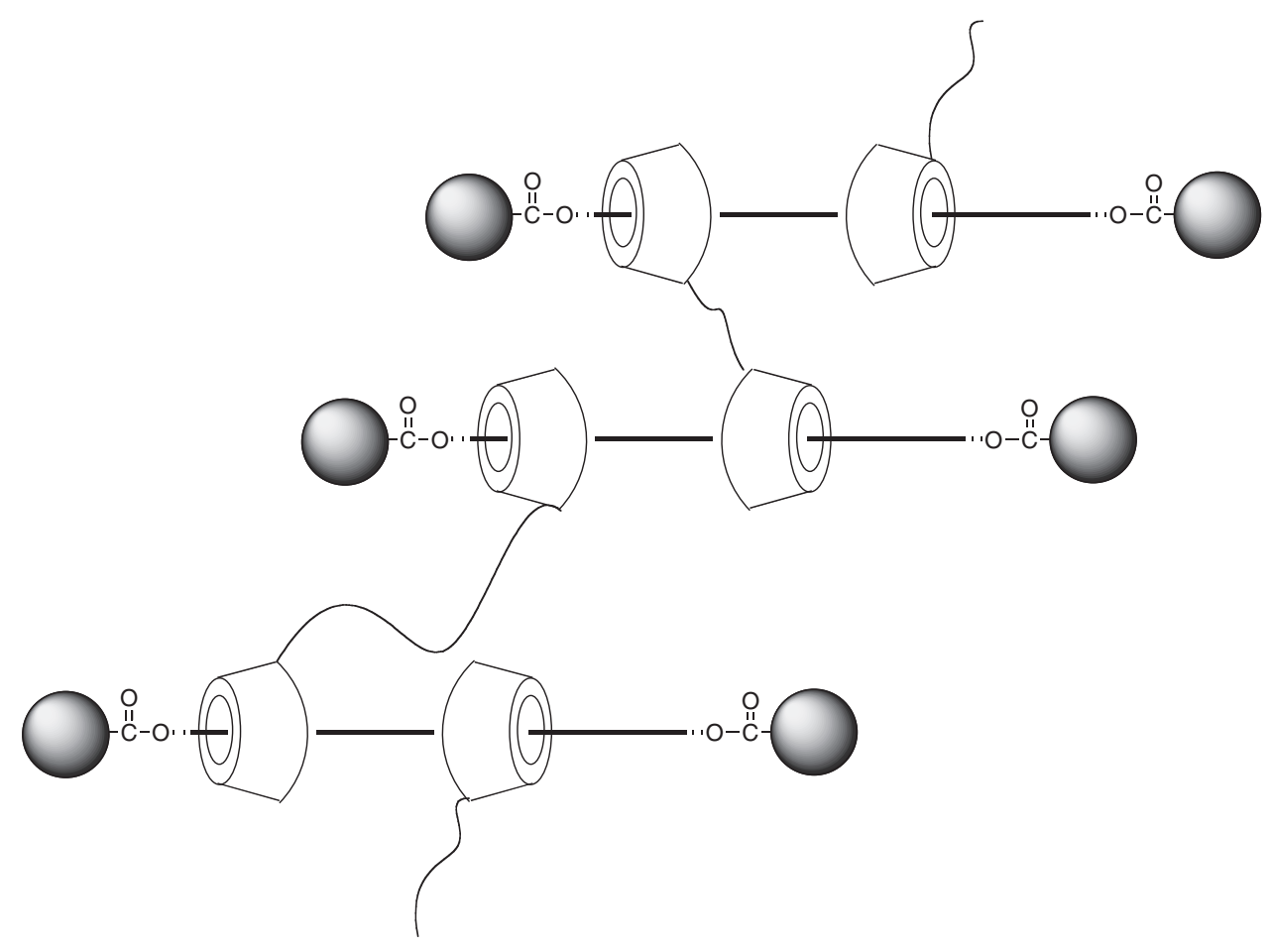

Scheme 17.

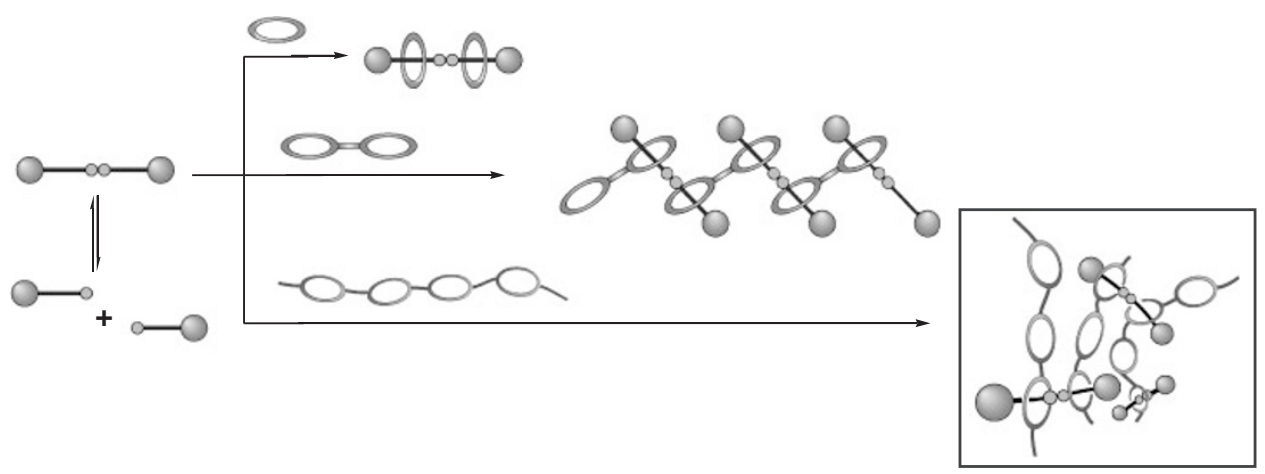

Scheme 18.

of $\alpha$-cyclodextrins and poly(ethylene glycol)s, in which the end-capping groups are linked by ester bonds on the assumption of in vivo nonenzymatic hydrolysis. They used the polyrotaxanes as crosslinkers for hydrophilic polymers such as poly(ethylene glycol) (Scheme 17). They linked intermolecularly $\alpha$ $\mathrm{CD}$ components of the polyrotaxanes with hydrophilic polymers to obtain hydrogels. The polyrotaxane hydrogels was more stable towards hydrolysis than the original polyrotaxanes. Furthermore, it was clearly shown that the time to reach complete erosion of the hydrogel was controllable by the polyrotaxane contents and the erosion profile was dominated over by the $M_{\mathrm{n}}$ of the main chain polymer.

Polyrotaxane Network Synthesized on the Basis of Dynamic Covalent Bond Chemistry

As mentioned previously, reversible cleavage of disulfide bond by thiol is quite useful for construction of topological linkage. [2]Rotaxane and [3]rotaxane were successfully obtained in high yields only by mixing a dumbbell-shaped axle and crown ether wheel in the presence of a catalytic amount of a thiol. ${ }^{21,22}$ Further, use of homoditopic crown ether resulted in the efficient formation of poly[3]rotaxane, the first topological poly[3]rotaxane, emphasizing the usefulness of the concept of dynamic covalent bond chemistry. ${ }^{30}$ If polyfunctional crown ether wheel is employed, polyrotaxane network should be formed according to Scheme 18.

Poly(crown ether) $\left(M_{\mathrm{n}} 5100, M_{\mathrm{w}} / M_{\mathrm{n}} 7.1\right)$, prepared according to Scheme 19, was mixed with a dumbbell-shaped disulfide with two ammonium salt moieties and a small amount of benzenethiol at $50^{\circ} \mathrm{C}$ (Scheme 20). After $20 \mathrm{~h}$, a gelled product was formed quantitatively. ${ }^{49}$ The swelled gel, which was original- 


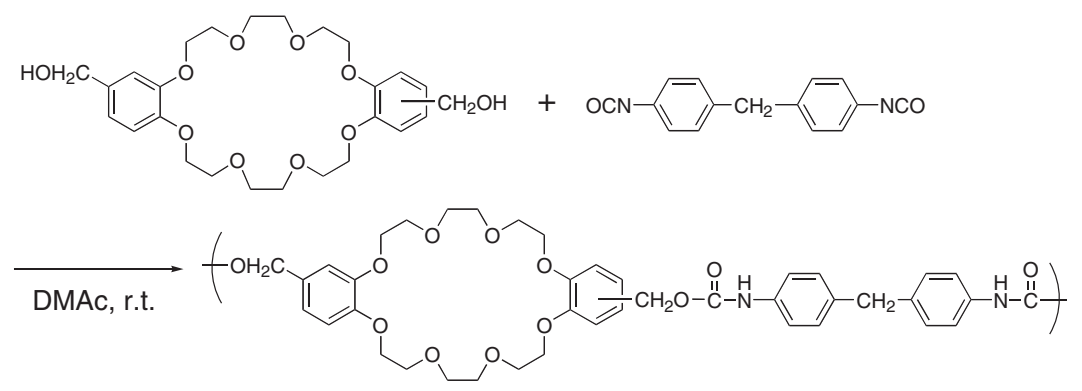

Scheme 19.<smiles>CC(C)=PCc1ccc(OCCOCCC(C)(C)C)c(OCCOCCOCCOc2cc(C)ccc2OCCOCCOCCOc2ccc(CC(C)(C)O)cc2Cc2cc(Br)cc(Br)c2)c1</smiles><smiles>[R]C#CCNc1ccc(Cc2ccc(NC(=O)CC)cc2)cc1</smiles><smiles>SCC[NH2+]Cc1cc(Br)cc(Br)c1</smiles>

Scheme 20.

ly elastic and transparent, was dried under reduced pressure to form a hard solid with plasticity. The result suggests that the gelation arises from crosslinking, especially from the formation of the [3] rotaxane-like structure composed of the dumbbell-shaped disulfide and the crown ether units, as shown in Scheme 20. The formation of the mechanical linkage as the crosslinking structure was confirmed by the detailed ${ }^{1} \mathrm{H}$ NMR studies of the gelled product as well as the model compounds. ${ }^{49}$

The gelled product swelled well in $N, N$-dimethylformamide (DMF; percentage swelling 1400\%) and dimethyl sulfoxide (DMSO; percentage swelling $840 \%$ ). But in methanol, the gel hardly swelled (percentage swelling 27\%). Figure 8 depicts two pictures of the gel before and after swelling in DMF. Although dry gel was rather hard and plastic, swelled gel was

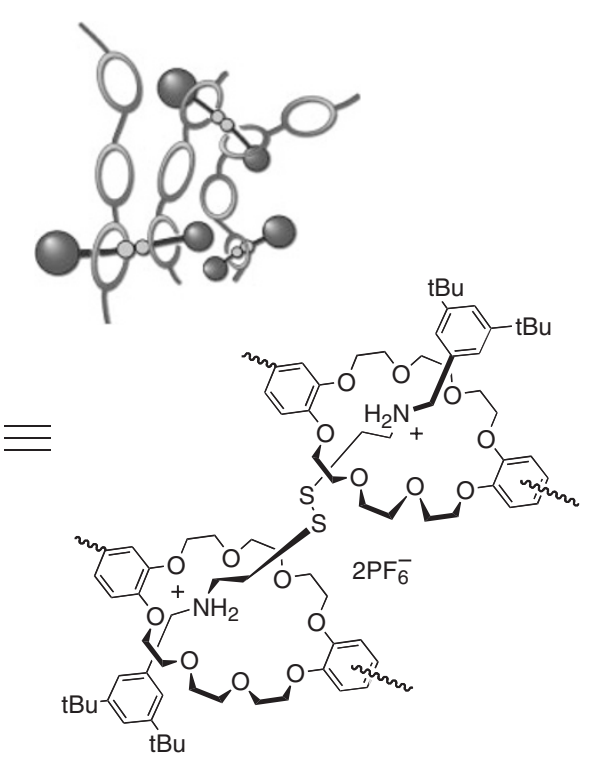



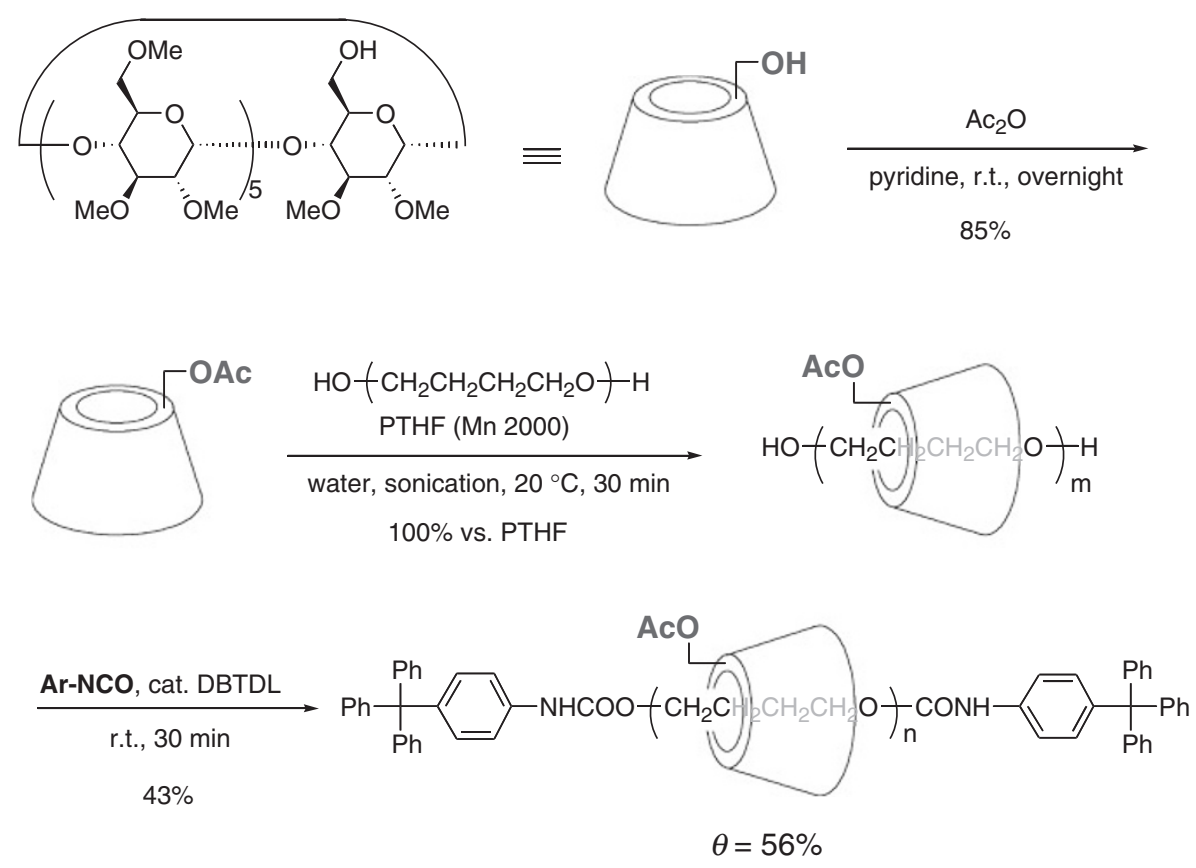

Scheme 21.

\section{Cyclodextrin-based Polyrotaxane Network}

Cyclodextrin is one of the useful wheel components of rotaxane and polyrotaxane. ${ }^{1-8}$ Takata and coworkers have recently developed a well-defined polyrotaxane network consisting of cyclodextrin wheel, ${ }^{50}$ like that of Ito's. ${ }^{44}$ Mono-hydroxy derivative of permethylated $\alpha$-cyclodextrin was prepared by the literature method with slight modification. ${ }^{51-53}$ Protection of the hydroxy group by acetylation was followed by complexation with PTHF $\left(M_{\mathrm{n}} 2000\right)$ in water to produce pseudopolyrotaxane (100\% yield), as depicted in Scheme 21. The key step is the end-capping which prevents the dethreading of PTHF from the wheel cyclodextrin. The attempts of the acylative end-capping of the pseudopolyrotaxane using a bulky isocyanate in solution were all in vain. However, the solid-state end-capping only by grinding a mixture of them in a mortar gave rise to the formation of polyrotaxane in which 8.2 wheels per axle on average were threaded (43\% yield, degree of coverage 56\%) (Scheme 21). ${ }^{50}$

The methanolysis of acetyl group on the wheel of the polyrotaxane quantitatively yielded hydroxy-functionalized polyrotaxane (Scheme 22). The polyrotaxane was treated with diphenylmethane diisocyanate $([\mathrm{NCO}] /[\mathrm{OH}]=0.5)$ in the presence of dibutyltin dilaurate (DBTDL) as catalyst in chloroform. The gellation immediately took place to quantitatively give gelled material, that is, the polymer crosslinked via interlocked bonding. The product gel showed remarkable swelling property in spite of a fairly high crosslinking ratio ( $\mathrm{ca}$. 50\%): the maximal absorption of typical organic solvents was $1.80 \mathrm{~g} / \mathrm{g}$ for DMF and $19.3 \mathrm{~g} / \mathrm{g}$ for chloroform, respectively. ${ }^{50}$ The gel is characterized not only by the topological crosslinking but also by the discrete crosslinked structure.

\section{Reversible Crosslinking-Decrosslinking: Recycle of Polyrotaxane Network}

Since the crosslinking is a reversible process, the starting materials can be perfectly recovered, if the crosslinked polymer is treated under appropriate conditions. To confirm this proposition, the crosslinked polymer was treated with a thiol which is a reduced form of the disulfidic axle instead of benzenethiol in the decrosslinking in DMF. The mixture became a colorless homogeneous solution after $70 \mathrm{~min}$ at $60^{\circ} \mathrm{C}$ (Scheme 23). Precipitation in methanol gave poly(crown ether) quantitatively by filtration, while a mixture of the axle and thiol was recovered in 92\% yield by the precipitation of the filtrate in water. Thus, there has been demonstrated a new concept for recyclable crosslinked polymer based on the reversible mechanical bonding characteristic of the rotaxane system. ${ }^{49}$

Takata et al. demonstrated a novel reversible crosslinking-decrosslinking system directed towards a new concept for the recycling of crosslinked polymers by utilizing a polyrotaxane network (Scheme 24). ${ }^{49}$ In this system, no apparent chemical bond formation or breaking takes place because the reversible disulfide bond cleavage occurring in the axle component is the only reaction during the crosslinking and decrosslinking in which a polyrotaxane network is formed and disassembled. Therefore, this protocol provides a novel concept for effective recycling: that is, the trunk polymer undergoes both crosslinking 


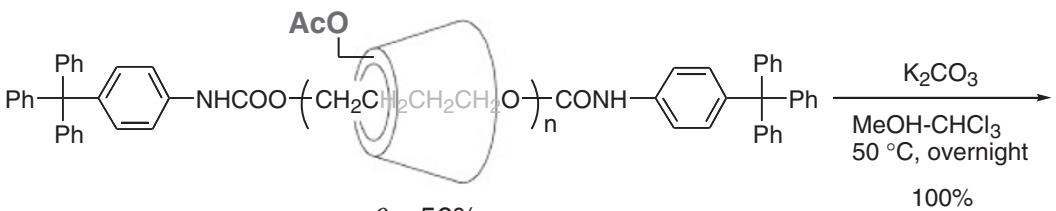

$$
\theta=56 \%
$$

$100 \%$
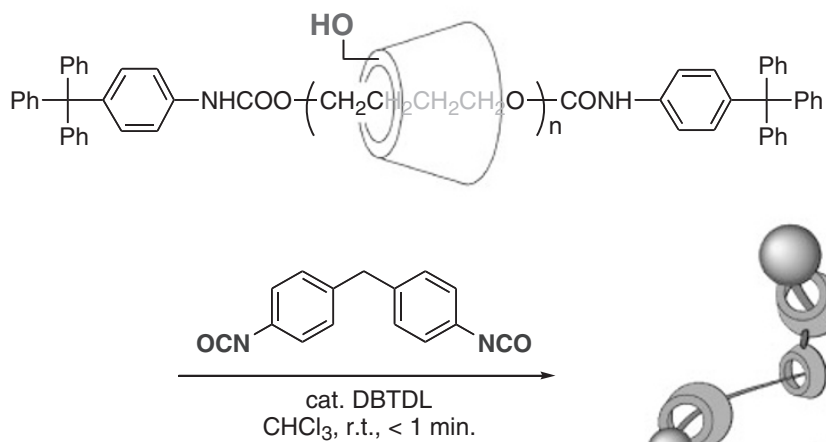

$100 \%$

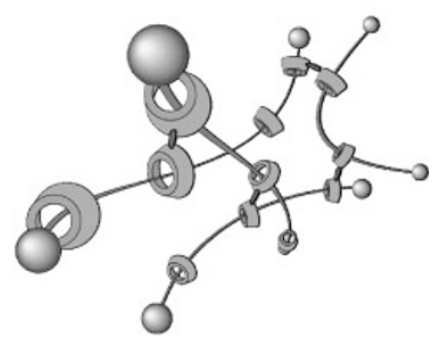

Scheme 22.
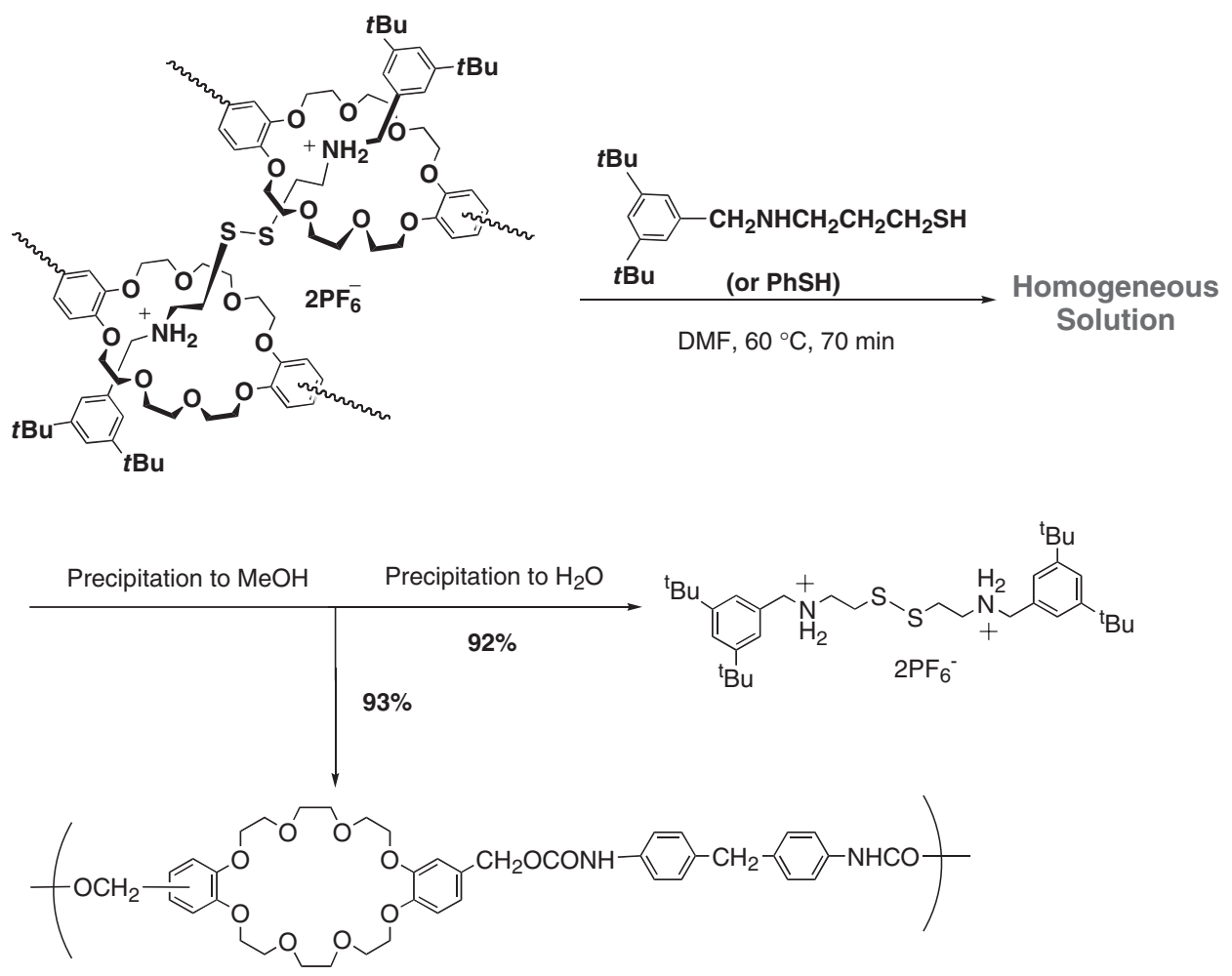

Scheme 23.

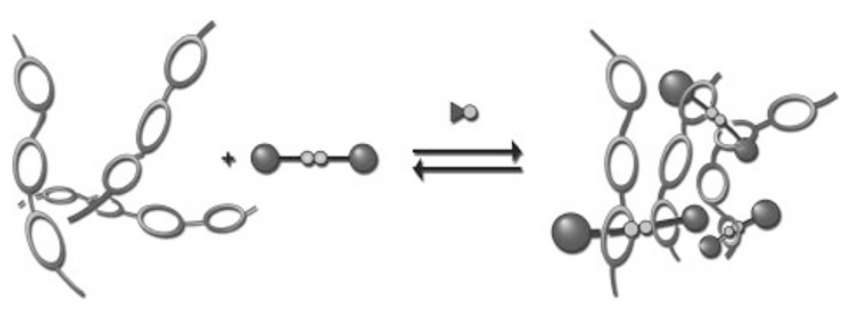

Scheme 24. and decrosslinking without receiving any injury because it does not participate directly in either of the two reactions.

In relation to recycle use of crosslinked polymer, Endo et al. reported the reversible crosslinkingdecrosslinking of a few polymers ${ }^{54-56}$ based on the equilibrium system between a spiroorthoester and its monoring-opened structure. ${ }^{57-59}$ When bifunctional spiroorthoester was treated with trifluoroacetic acid 

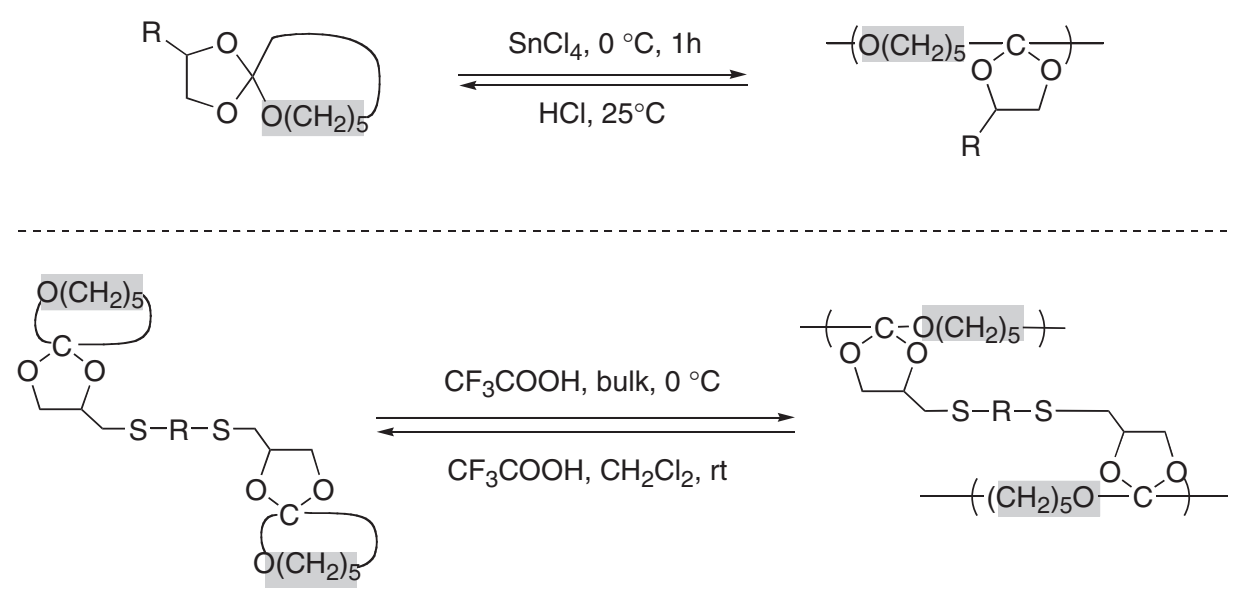

Scheme 25.

at low temperature without solvent, the corresponding crosslinked polymer was obtained in a quantitative yield (Scheme 25). ${ }^{54}$ On the other hand, the crosslinked polymer was easily changed to soluble starting material by reacting with trifluoroacetic acid in dichloromethane at room temperature. Similarly, as shown in Scheme 26, side-chain type poly(spiroorthoester)-co-polyacrylonitrile gave a quantitative yield of the crosslinked polymer by the catalysis with trifluoroacetic acid at high concentration at $-10{ }^{\circ} \mathrm{C} .{ }^{55,56}$ When the crosslinked polymer was treated with trifluoroacetic acid at lower concentration and higher temperature, the starting soluble polymer was obtained in 97\% yield. The GPC change by crosslinking showed no appreciable difference (Scheme 26).

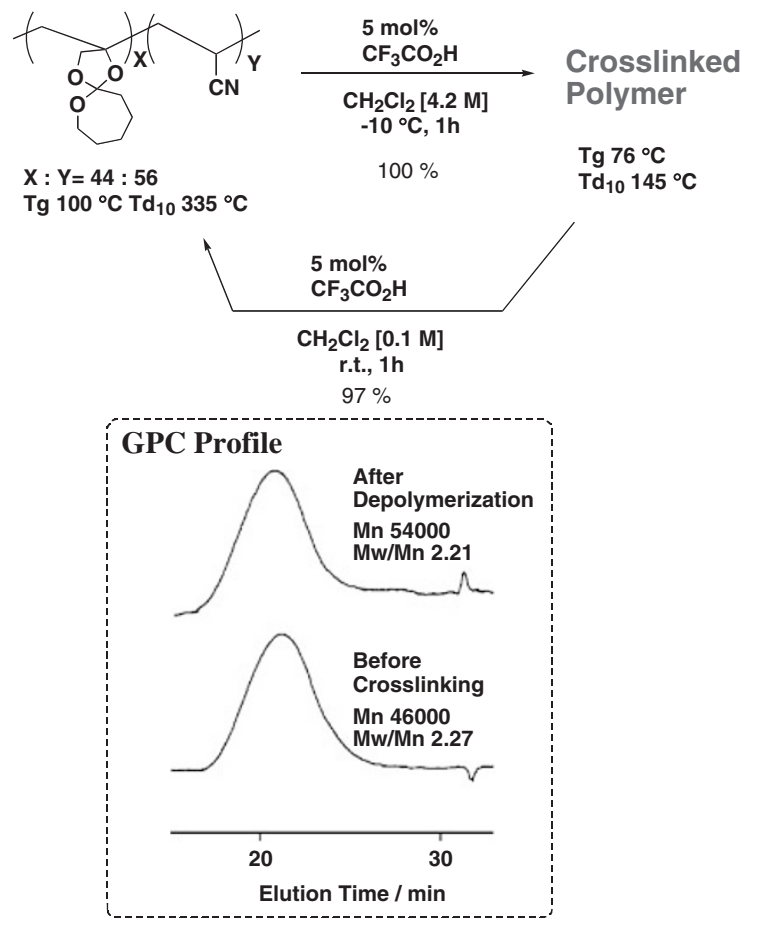

Scheme 26.
Thus, the reversible crosslinking-decrosslinking system may strongly contribute to the development of novel recyclable crosslinked polymers and the concept of recycle use of crosslinked polymer.

\section{CONCLUDING REMARKS}

This article has dealt with subjects mainly concerning with synthesis of rotaxane, polyrotaxane, and polyrotaxane network which are constructed on the basis of the concept of the dynamic covalent bond chemistry. The concept is simple and quite suitable for the application to the molecular integration in supramolecular and supramacromolecular chemistry. Reversible nature of the dynamic covalent bond further displays wide applicability in many fields such as the recycle of crosslinked polymer. The crosslinking reaction between poly(crown ether) and a dumbbell-shaped disulfidic bisammonium axle afforded a transparent elastic gel probably possessing a potential application. Further studies will provid useful materials derived from polyrotaxanes consisting of topological monomer linkages in the main chain.

Meanwhile, there is an interesting interlocked polymer possibly possessing novel property: polycatenane or $[n]$ catenane consisiting only of ring components. Polycatenane has long been the biggest target polymer that has never been synthesized so far. Kihara and Takata has recently approached to polycatenane by applying the Diels-Alder protocol to A-B type bifuncational [2]catenane monomer which has both diene and dienophile functionalities in their each ring to obtain "bridged polycatenane" (Scheme 27) ${ }^{60}$ In relation to polycatenane, Endo et al. have reported the possible crosslinking by polycatenane formation in the polymer derived by the ring opening polymerization of cyclic disulfides. ${ }^{61}$ Much attention has focused on the synthesis and property of polycatenane and polycatenane-based materials. 


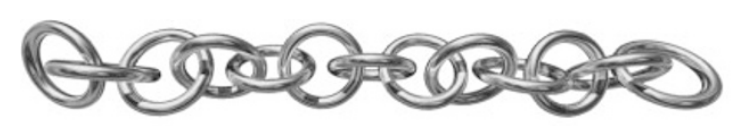

Polycatenane ([n]Catenane)

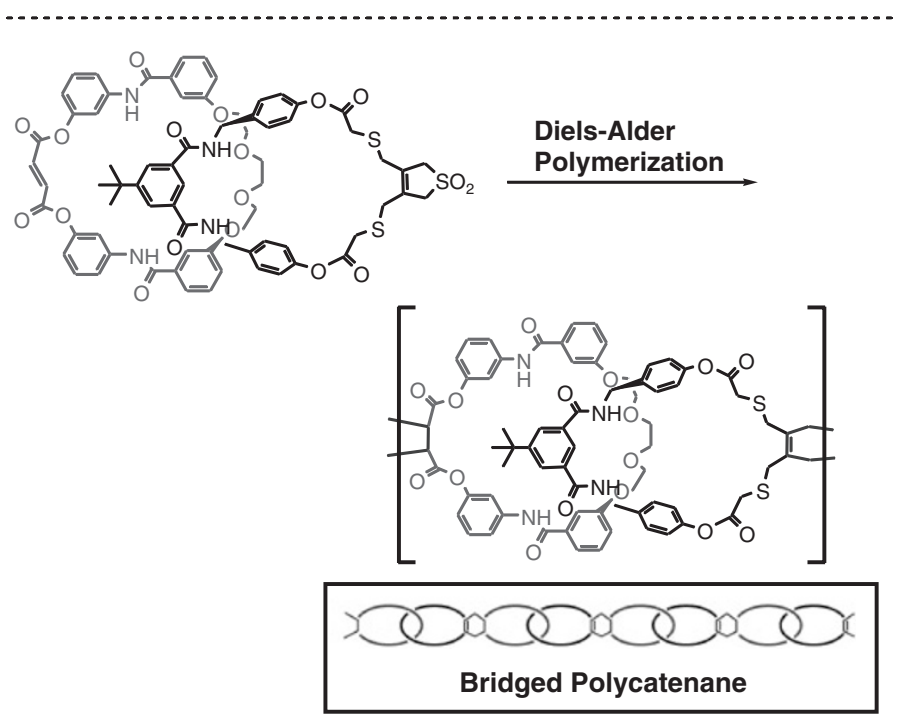

Scheme 27.

Thus, the interlocked polymers have great potentiality as novel materials which will be realized in the near future.

\section{REFERENCES}

1. J.-P. Sauvage and C. Dietrich-Buchecker, "Molecular Catenanes, Rotaxanes, and Knots," Wiley-VCH, New York, Weinheim, 1999.

2. D. B. Amabilino and J. F. Stoddart, Chem. Rev., 95, 2725 (1995).

3. T. Takata and N. Kihara, Rev. Heteroatom Chem., 22, 197 (2000).

4. N. Kihara and T. Takata, Yukigosei Kagaku Kyukaishi, 59, 206 (2001).

5. H. W. Gibson, M. C. Bheda, and P. T. Engen, Prog. Polym. Sci., 19, 843 (1994).

6. F. M. Raymo and J. F. Stoddart, Chem. Rev., 99, 1643 (1999).

7. A. Harada, Acta Polym., 49, 3 (1998).

8. T. Takata, N. Kihara, and Y. Furusho, Adv. Polym. Sci., 171, 1 (2004).

9. A. G. Kolchinski, D. H. Busch, and N. W. Alcock, J. Chem. Soc., Chem. Commun., 1289 (1995).

10. P. R. Ashton, E. J. T. Chrystal, P. T. Glink, S. Menzer, C. Schuavo, N. Spencer, J. F. Stoddart, P. A. Tasker, A. J. P. White, and D. J. Williams, Chem. Eur. J., 2, 709 (1996).

11. H. Kawasaki, N. Kihara, and T. Takata, Chem. Lett., 10, 1015 (1999).

12. N. Kihara, N. Nakakoji, and T. Takata, Chem. Lett., 924 (2002).

13. A. G. Kolchinski, N. W. Alcock, R. A. Roesner, and D. H. Busch, Chem. Commun., 1437 (1998).

14. N. Kihara, J.-I. Shin, Y. Ohga, and T. Takata, Chem. Lett., 6,
592 (2001).

15. P. R. Ashton, P. T. Glink, J. F. Stoddart, S. Menzer, P. A. Tasker, A. J. P. White, and D. J. Williams, Tetrahedron Lett., 37, 6217 (1996).

16. P. R. Ashton, P. T. Glink, J. F. Stoddart, P. A. Tasker, A. J. P. White, and D. J. Williams, Chem. Eur. J., 2, 729 (1996).

17. N. Watanabe, T. Yagi, N. Kihara, and T. Takata, Chem. Commun., 2720 (2002).

18. T. Takata, H. Kawasaki, S. Asai, N. Kihara, and Y. Furusho, Chem. Lett., 2, 111 (2000).

19. T. Takata, H. Kawasaki, N. Kihara, and T. Furusho, Macromolecules, 34, 5449 (2001).

20. a) S. J. Rowan, S. J. Cantrill, G. R. L. Cousins, J. K. M. Sanders, and J. F. Stodddart, Angew. Chem. Int. Ed., 41, 898 (2002).

b) S. J. Rowan, S. J. Cantrill, and J. F. Stoddart, Org. Lett., 1, 129 (1999).

c) S. J. Rowan and J. F. Stoddart, J. Am. Chem. Soc., 122, 164 (2000).

21. Y. Furusho, T. Hasegawa, A. Tsuboi, N. Kihara, and T. Takata, Chem. Lett., 1, 18 (2000).

22. Y. Furusho, T. Oku, T. Hasegawa, A. Tsuboi, N. Kihara, and T. Takata, Chem. Eur. J., 9, 2895 (2003).

23. Y. Furusho, T. Oku, G. A. Rajkumar, and T. Takata, Chem. Lett., 33, 52 (2004).

24. For example, Y. Geerts, D. Muscat, and K. Müllen, Macromol. Chem. Phys., 196, 3425 (1995).

25. S. J. Rowan, S. J. Cantrill, J. F. Stoddart, A. J. P. White, and D. J. Williams, Org. Lett., 2, 759 (2000).

26. M. C. Jiménez, C. Dietrich-Buchecker, and J.-P. Sauvage, Angew. Chem., Int. Ed., 39, 3284 (2000).

27. T. Hoshino, M. Miyauchi, Y. Kawaguchi, H. Yamaguchi, and A. Harada, J. Am. Chem. Soc., 122, 9876 (2000).

28. T. Fujimoto, Y. Sakata, and T. Kaneda, Chem. Comunn., 
2143 (2000).

29. H. Onagi, C. J. Easton, and S. F. Lincoln, Org. Lett., 3, 1041 (2001).

30. T. Oku, Y. Furusho, and T. Takata, J. Polym. Sci., Part A: Polym. Chem., 41, 119 (2003).

31. N. Yamaguchi and H. W. Gibson, Angew. Chem., Int. Ed., 38, 143 (1999).

32. Y. Sohgawa, H. Fujimori, J. Shoji, Y. Furusho, N. Kihara, and T. Takata, Chem. Lett., 774 (2001).

33. W. J. Lee and G. M. Whiteside, J. Org. Chem., 58, 642 (1993) and references cited therein.

34. C. Gong and H. W. Gibson, Macromol. Chem. Phys., 199, 1801 (1998).

35. Y. Delaviz and H. W. Gibson, Macromolecules, 25, 4859 (1992).

36. H. W. Gibson, D. S. Nagveker, J. Powell, C. Gong, and W. S. Bryant, Tetrahedron, 53, 15197 (1997).

37. C. Gong and H. W. Gibson, J. Am. Chem. Soc., 119, 8585 (1997).

38. C. Gong and H. W. Gibson, J. Am. Chem. Soc., 119, 5862 (1997).

39. A. Zada, Y. Avny, and A. Zilkha, Eur. Polym. J., 35, 1159 (1999).

40. A. Zada, Y. Avny, and A. Zilkha, Eur. Polym. J., 36, 351 (2000).

41. A. Zada, Y. Avny, and A. Zilkha, Eur. Polym. J., 36, 359 (2000).

42. H. Oike, T. Mouri, and Y. Tezuka, Macromolecules, 34, 6229 (2001).

43. M. Kubo, T. Hibino, M. Tamura, T. Uno, and T. Itoh, Macromolecules, 35, 5816 (2002).

44. Y. Okumura and K. Ito, Adv. Mater., 13, 485 (2001).

45. J. Araki, C. Zhao, and K. Ito, Macromolecules, 38, 7524 (2005).
46. J. Watanabe, T. Ooya, and N. Yui, J. Biomater. Sci., Polym. Ed., 10, 1275 (1999).

47. J. Watanabe, T. Ooya, K. D. Park, Y. H. Kim, and N. Yui, J. Biomater. Sci., Polym. Ed., 11, 1333 (2000).

48. T. Ichi, J. Watanabe, T. Ooya, and N. Yui, Biomacromolecules, 2, 204 (2001).

49. T. Oku, Y. Furusho, and T. Takata, Angew. Chem., Int. Ed., 43, 966 (2004).

50. N. Kihara, K. Hinoue, and T. Takata, Macromolecules, 38, 223 (2005).

51. L. D. Melton and K. N. Slessor, Carbohydr. Res., 18, 29 (1971).

52. M. Tanaka, Y. Kawaguchi, T. Niinae, and T. Shono, J. Chromotogr., 314, 193 (1984).

53. T. Kaneda, T. Fujimoto, J. Goto, K. Asano, Y. Yasufuku, J. H. Jung, C. Hosono, and Y. Sakata, Chem. Lett., 5, 514 (2002).

54. T. Endo, T. Suzuki, F. Sanda, and T. Takata, Macromolecules, 29, 3315 (1996).

55. T. Endo, T. Suzuki, F. Sanda, and T. Takata, Macromolecules, 29, 4819 (1996).

56. T. Endo, T. Suzuki, F. Sanda, and T. Takata, Bull. Chem. Soc. Jpn., 70, 1205 (1997).

57. S. Chikaoka, T. Takata, and T. Endo, J. Polym. Sci., Part A: Polym. Chem., 28, 3103 (1990).

58. S. Chikaoka, T. Takata, and T. Endo, Macromolecules, 24, 331 (1991).

59. S. Chikaoka, T. Takata, and T. Endo, Macromolecules, 24, 6557 (1991).

60. N. Watanabe, Y. Ikari, N. Kihara, and T. Takata, Macromolecules, 37, 6663 (2004).

61. K. Endo, T. Shiroi, N. Murata, G. Kojima, and T. Yamanaka, Macromolecules, 37, 3143 (2004).

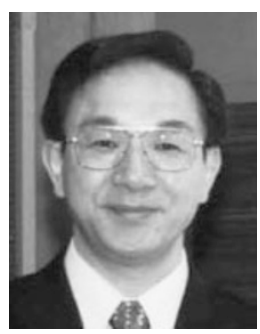

Toshikazu Takata was born in Toyama Prefecture, Japan in 1953. He received his B. S. degree in 1976 from Okayama University and $\mathrm{Ph}$. D. degree in 1981 from Tsukuba University under the supervision of Professor Shigeru Oae. After joining Professor Wataru Ando's group as a research associate in Tsukuba University for a few years, he moved to Tokyo Institute of Technology in 1985 (Professor Takeshi Endo's group, Chemical Resources Laboratory), where he started his career as a polymer chemist in the field of polymer synthesis. He was promoted to associate professor in 1988 at the same institution and full professor in 1994 at Japan Advanced Institute of Science and Technology, where synthesis of novel artificial helical molecules was one of his research interests. On the occasion of his movement to Osaka Prefecture University in 1995, he started to study the supramolecular chemistry and macromolecular chemistry of interlocked molecules such as (poly)rotaxanes. Present research interests of Professor Takata are divided into four areas: (1) Supramolecular and macromolecular chemistry of (poly)rotaxane and (poly)catenane; (2) Synthesis and application of artificial helical molecules; (3) Synthesis of high performance polymers; (4) Development of novel materials and processes compatible to environment. He was the recipient of several awards, e.g., The Ichimura Prize (2002) and The Award of the Society of Polymer Science, Japan (2004). He is the author and coauthor of $c a .300$ original papers, $c a .80$ reviews and books, and $c a .60$ patents. 